\title{
Exceptional field theory: SL(5)
}

\section{Edvard T. Musaev}

National Research University Higher School of Economics, Faculty of Mathematics, 7, st. Vavilova, 117312 Moscow, Russia

Max-Planck-Institut für Gravitationsphysik (Albert-Einstein-Institut), Am Mühlenberg 1, 14476 Potsdam, Germany

E-mail: edvard.musaev@aei.mpg.de

ABSTRACT: In this work the exceptional field theory formulation of supergravity with SL(5) gauge group is considered. This group appears as a U-duality group of $D=7$ maximal supergravity. In the formalism presented the hidden global duality group is promoted into a gauge group of a theory in dimensions $7+$ number of extended directions. This work is a continuation of the series of works for $E_{8,7,6}, \mathrm{SO}(5,5)$ and $\mathrm{SL}(3) \times \mathrm{SL}(2)$ duality groups.

KeYwords: Gauge Symmetry, String Duality, M-Theory

ARXiv EPRINT: 1512.02163 


\section{Contents}

1 Introduction 1

2 Supergravity in $D=7 \quad 3$

3 Tensor hierarchy and Bianchi identities 3

4 Covariant exceptional field theory $\quad 7$

4.1 Kinetic Lagrangian and invariant potential 8

$\begin{array}{lr}4.2 \text { Topological Lagrangian } & 9\end{array}$

$\begin{array}{ll}4.3 D=6+1 \text { diffeomorphisms } & 10\end{array}$

5 Embeddings of $\mathrm{D}=11$ and Type IIB supergravity 13

6 Discussion and outlook 16

$\begin{array}{ll}\text { A Notations and conventions } & 17\end{array}$

$\begin{array}{lr}\text { B The algebra of SL(5) } & 18\end{array}$

$\begin{array}{ll}\text { C Useful identities } & 19\end{array}$

$\begin{array}{ll}\text { D Gauge invariance of the topological Lagrangian } & 20\end{array}$

\section{Introduction}

The construction of Extended Geometry appeared in a series of works [1-6] and many others has got a lot of attention recent years in particular in the context of string cosmology $[7,8]$, searches for non-geometric solutions [9-16], generalized Scherk-Schwarz compactifications and embeddings of Type II solutions [17-24] and many others. The basic idea of the model is to turn the hidden U-duality symmetries of compactified (half-)maximal supergravities into a manifest gauge symmetry of a full non-compactified theory. This is achieved by introducing new coordinates in addition to the existing 10 or 11 . In the string or Mtheory interpretation these new coordinates correspond to winding modes of strings and D- or M-branes (see e.g. [2]). Geometric meaning of the extended space was investigated in the works [25-29]. Very important direction of research is bound to the question of finite coordinate transformations in the extended space. To the moment it is not clear how to integrate infinitesimal generalized diffeomorphisms to a large transformation, however there has been certain progress made [30-32]. In the work [33] attempts to construct the algebra without the need of section condition were made. Construction of superspace for DFT was considered in the work [34]. In the series of works [35-37] it was shown that the 
string and M-theoretical extended objects can be understood as wave-like or monopole-like solutions of Exceptional Field Theory.

Since the particular U-duality group $E_{n(n)}$ depends on the number of compact directions $n$ the full set of coordinates of EFT naturally splits into the so-called "external" space-time coordinates denoted by $x^{\mu}$ and "internal" extended coordinates denoted by $\mathbb{X}^{M}$. Here $\mu$ runs over all would-be non-compact directions, i.e. from 1 to $11-n$ (for the maximal case), while the capital Latin index labels the necessary representation of the U-duality group, that catches all the translational and winding modes of the M2 and M5 branes.

Although, EFT is formulated as a non-compactified theory, one has always keep in mind an additional condition, that forces us to either drop all or a subset of the extended coordinates returning effectively to the conventional supergravity or its toroidal compactifications, or to perform a generalized Scherk-Schwarz reduction, leading to the (half)maximal gauged supergravity [17-19, 21].

This condition, named section condition, appears in the algebra of generalized diffeomorphisms given by the so-called generalized Lie derivative [38]. These may be understood as local $\mathrm{U}(\mathrm{T})$-duality transformations and the theory should be (co)invariant with respect to them. For more details the reader is referred to the reviews [39-41].

Special properties of generalized Lie derivatives allow to obtain the tensor hierarchy of gauged supergravity as a natural consequence of the algebra and the section condition. It was proposed in $[42,43]$ to let all the gauge parameters to depend on the whole set of coordinates $\left(x^{\mu}, \mathbb{X}^{M}\right)$, and use the vector field $A_{\mu}^{M}$ of the corresponding supergravity to introduce a derivative covariant with respect to generalized Lie transformations. Due to failure of the Jacobi identity for these transformations one is required to deform the 2-form field strength in the spirit of tensor hierarchy introducing a 2-form potential. Bianchi identities uniquely determine the corresponding field strength and the sequence can be continued.

Based on these ideas is the Exceptional Field Theory formalism presented in the series of papers [44-48] for the groups $\mathrm{E}_{8,7,6}, \mathrm{SO}(5,5)$ and $\mathrm{SL}(3) \times \mathrm{SL}(2)$, that correspond to $D=3,4,5,6$ and 8 maximal supergravities. The supersymmetric construction that includes fermions is given in [49-51]. In this paper we aim at the construction for $D=7$ and the group SL(5). To the moment there has been large progress in investigation of the SL(5) extended geometry $[52,53]$. To be noted is the work [54] that considers the internal sector, i.e. the so-called scalar potential, for $\mathrm{SL}(\mathrm{N})$ group for any $N$. In the current paper we present the full EFT construction, including the gauge kinetic and topological sector.

This paper starts with the section 2, where the bosonic field content of the maximal supergravity in $D=7$ is discussed. In section 3 we briefly review the construction of exceptional field theory and tensor hierarchy to set up our notation for further reference. In section 4 we construct a duality covariant kinetic and topological Lagrangian and check its invariance under external diffeomorphisms. We comment there on the feature of EFT when all the numerical prefactors in the Lagrangian are fixed already at the bosonic level. Embedding of the 11-dimensional and Type II supergravities into the formalism is considered in section 5. Finally, in the appendix we present the notations used, conventions on the SL(5) algebra, provide some useful identities and explicitly check gauge invariance of the topological Lagrangian. 


\section{$2 \quad$ Supergravity in $D=7$}

Maximal ungauged supergravity in 7 dimensions with the global duality group SL(5) was constructed in [55]. The field content after the $7+4$ split is given by

$$
\left\{g_{\mu \nu}, A_{\mu a}, \phi_{a b}, C_{\mu \nu \rho}, B_{\mu \nu a}, A_{\mu a b}, \phi_{a b c}\right\},
$$

where the internal indices $a, b$ run from 1 to 4 . We have a total of ten 1 -form fields, $A_{\mu a}$ and $A_{\mu a b}$, which transform in the $\overline{\mathbf{1 0}}$ representation of the duality group SL(5) (see [56] for more details on how the supergravity fields are organized into irreps of U-duality group). We will denote this representation as $A_{\mu}^{M}, M=1, \ldots, 10$, where $M$ may also be thought of as a pair of SL(5) fundamental indices, $A_{\mu}^{M}=\frac{1}{2} A_{\mu}^{[i j]}, i, j=1, \ldots, 5$. More details on how we treat the doubled indices can be found in the appendix A.

The duality relation between 3 -forms and 2 -forms in seven space-time dimensions allows to collect the four fields $B_{\mu \nu a}$ and the 3 -form $C_{\mu \nu \rho}$ together, resulting in five 2-form fields $B_{\mu \nu i}$ transforming in the fundamental representation of $\mathrm{SL}(5)$.

There are now 14 scalar fields, $\phi_{a b}$ and $\phi_{a b c}$, whose dynamics may be formulated in terms of the matrix $V_{i}^{\alpha \beta}$ parameterizing the coset $\mathrm{SL}(5) / \mathrm{SO}(5)$. The small Greek indices $\alpha, \beta=1, \ldots, 4$ label the fundamental representation of $\operatorname{USp}(4) \simeq \operatorname{Spin}(5)$. We require the scalar matrix $V_{i}^{\alpha \beta}$ to be antisymmetric in $\alpha, \beta$ and traceless with respect to the $\operatorname{USp}(4)$ invariant tensor $\Omega_{\alpha \beta}, V_{i}^{\alpha \beta} \Omega_{\alpha \beta}=0$ [57]. These constraints cut the number of degrees of freedom of $V_{i}^{\alpha \beta}$ down to 25 . Imposing additionally that $\operatorname{det} V=1$ we constrain $V$ to have the right number of degrees of freedom and to be an SL(5) element. To respect the tracelessness condition, the inverse of $V_{i}^{\alpha \beta}$ is defined by the following identities

$$
V_{i}^{\alpha \beta} V_{\alpha \beta}^{j}=\delta_{i}^{j}, \quad V_{i}^{\alpha \beta} V_{\gamma \delta}^{i}=\delta_{\gamma \delta}^{\alpha \beta}-\frac{1}{4} \Omega^{\alpha \beta} \Omega_{\gamma \delta} .
$$

One defines the $\mathrm{SL}(5)$ generalized metric as $m_{i j}=V_{i}^{\alpha \beta} V_{j \alpha \beta}$, but sometimes it will be convenient to use the generalized metric in the $\mathbf{1 0}$ of $\mathrm{SL}(5)$, defined as

$$
\mathcal{M}_{M N} \Longrightarrow \mathcal{M}_{i j, k l}=m_{i k} m_{j l}-m_{i l} m_{j k} .
$$

As in the $\mathrm{SO}(5,5)$ case, duals for the 2-forms must be introduced as independent fields, in order to facilitate the description of different possible gaugings. Thus we introduce a set of 3 -form fields $C_{\mu \nu \rho}{ }^{i}$, transforming in the $\overline{\mathbf{5}}$ of $\mathrm{SL}(5)$. These are related by a duality condition that will arise as an equation of motion. It will be convenient to redefine the 2and the 3 -form fields with the indices labeling the $\overline{\mathbf{1 0}}$ of SL(5), $B_{\mu \nu}{ }^{K L}$ and $C_{\mu \nu \rho}{ }^{N, K L}$ :

$$
\begin{aligned}
B_{\mu \nu i} & =2 \epsilon_{i k l m n} B_{\mu \nu}{ }^{k l m n}, \\
C_{\mu \nu \rho}^{m} & =-6 \epsilon_{n k l r s} C_{\mu \nu \rho}^{m n, k l r s} .
\end{aligned}
$$

\section{Tensor hierarchy and Bianchi identities}

Let us now briefly review the EFT construction and setup our conventions. Transformation of covariant objects in Exceptional Field Theory is defined by the usual rule

$$
\delta_{\Lambda} V^{M}=\left(\mathcal{L}_{\Sigma} V\right)^{M}=\left(L_{\Lambda} V\right)^{M}+Y_{K L}^{M N} \partial_{N} \Lambda^{K} V^{L} \equiv[\Lambda, V]_{D}^{M},
$$


where $[,]_{D}$ denotes the Dorfman bracket. For the SL(5) U-duality group the $Y$-tensor is given by

$$
Y_{P Q}^{M N}=\epsilon^{m M N} \epsilon_{m P Q}
$$

where $\epsilon^{m M N}$ is an SL(5) invariant tensor whose components are given by the alternating symbol $\epsilon^{m k l p q}$. Note, that each large Latin letter parametrizes 10 representation of SL(5) and through the paper is always equivalent to a pair of small Latin indices parameterizing $\mathbf{5}$ of SL(5) (see appendix A).

Since the $Y$-tensor is related to the projector on the adjoint it is straightforward to write the generalized Lie derivative of a generalized vector in the following form

$$
\delta_{\Lambda} V^{M}=\left(\mathcal{L}_{\Lambda} V\right)^{M}=\Lambda^{N} \partial_{N} V^{M}-3 \mathbb{P}^{M}{ }_{L}{ }^{N}{ }_{K} \partial_{N} \Lambda^{K} V^{L}+\frac{1}{5}\left(\partial_{K} \Lambda^{K}\right) V^{M} .
$$

Here the last term plays the role of a weight term, that could be in principle added to any transformation. However, here it directly follows from the algebra. Rewriting the projector $\mathbb{P}$ explicitly as in the appendix B one obtains the following transformation of a field in the fundamental $\mathbf{5}$ representation

$$
\left(\mathcal{L}_{\Lambda} U\right)^{m}=\Lambda^{N} \partial_{N} U^{m}-\frac{1}{4}\left(t_{n}^{m}\right)_{p q}^{k l} \partial_{k l} \Lambda^{p q} U^{n}+\frac{1}{10}\left(\partial_{K} \Lambda^{K}\right) U^{m} .
$$

The weight $\lambda\left(U^{m}\right)=1 / 10$ is a half of that for $V^{m n}$ as it should be since one may always introduce a tensor $U^{[m} U^{n]}$ in $\mathbf{1 0}$ that has the weight $2 \lambda\left(U^{m}\right)$. In what follows it will prove useful to have transformation rules for the tensor $B_{m}=\epsilon_{m K L} B^{K L}$ obtained by contraction of a 2-rank generalized tensor of weight $\lambda\left(B^{K L}\right)=2 / 5$ with the epsilon-tensor. The resulting generalized tensor belongs to the $\overline{\mathbf{5}}$ representation and transforms as

$$
\left(\mathcal{L}_{\Lambda} B\right)_{m}=\Lambda^{N} \partial_{N} B_{m}+\frac{1}{4}\left(t^{n}{ }_{m}\right)_{p q}^{k l} \partial_{k l} \Lambda^{p q} B_{n}+\frac{2}{5}\left(\partial_{K} \Lambda^{K}\right) B_{m}
$$

As expected, this differs from the above transformation for a tensor in $\mathbf{5}$ only by the weight term.

It is important to mention the section condition $Y_{K L}^{M N} \partial_{M} \otimes \partial_{N}=0$, that for the case of SL(5) U-duality group can be written as

$$
\epsilon^{i m n k l} \partial_{m n} \otimes \partial_{k l}=0
$$

In this form the section condition implies existence of trivial generalized transformation given by $\Lambda_{0}^{m n}=\epsilon^{m n k l p} \partial_{k l} \xi_{p}$, i.e. $\delta_{\Lambda_{0}} V^{M}=0$ up to the section condition.

The $E$-bracket is introduced in the usual way via commutation of generalized Lie derivative

$$
\left[\mathcal{L}_{\Lambda_{1}}, \mathcal{L}_{\Lambda_{2}}\right]=\mathcal{L}_{\left[\Lambda_{1}, \Lambda_{2}\right]_{E}}
$$

and reads

$$
\left[\Lambda_{1}, \Lambda_{2}\right]_{E}=\left[\Lambda_{1}, \Lambda_{2}\right]_{D}-\frac{1}{2} Y_{K L}^{M N} \partial_{N}\left(\Lambda_{1}^{K} \Lambda_{2}^{L}\right) .
$$

Hence, the E-bracket is antisymmetric while the Dorfman bracket is not. Finally, we mention following Jacobi identity for the $E$-bracket

$$
\left[\left[\Lambda_{[1}, \Lambda_{2}\right]_{E}, \Lambda_{3]}\right]_{E}^{M}=\frac{1}{6} Y_{K L}^{M N} \partial_{N}\left(\left[\Lambda_{[1}, \Lambda_{2}\right]_{E}^{K} \Lambda_{3]}^{L}\right) .
$$


This failure of the Jacobi identity and lack of antisymmetric property of the $D$-bracket naturally leads to tensor hierarchy in EFT. In other words, tensor fields of higher ranks naturally appear to preserve covariance of expressions.

The long space-time derivative, covariant with respect to the D-bracket is defined in the usual way

$$
\mathcal{D}_{\mu}=\partial_{\mu}-\mathcal{L}_{A_{\mu}}=\partial_{\mu}-\left[A_{\mu}, \bullet\right]_{D},
$$

with the generalized vector field $A_{\mu}^{M}$ transforming as

$$
\delta_{\Lambda} A_{\mu}^{M}=\partial_{\mu} \Lambda^{M}-\left[A_{\mu}, \Lambda\right]_{D}^{M}=\mathcal{D}_{\mu} \Lambda^{M}
$$

Note, that since D- and E-brackets differ by a trivial transformation (see (3.8)) the above choice is matter of convention. The transformation in this form is taken to keep analogy with the conventional Yang-Mills construction.

As usual, the commutator of covariant derivatives defines the field strength of the gauge field that fails to be covariant, so one introduces a 2-form gauge field $B_{\mu \nu} K L$ whose degrees of freedom are identified with those of the 2 -form field $B_{\mu \nu m}$ via

$$
B_{\mu \nu m}=8 \epsilon_{m M N} B_{\mu \nu}^{K L}=2 \epsilon_{m p q r s} B_{\mu \nu}^{p q r s} .
$$

As a result one has the following tensor hierarchy [47]

$$
\begin{aligned}
{\left[\mathcal{D}_{\mu}, \mathcal{D}_{\nu]}\right]=} & -\mathcal{L}_{\mathcal{F}_{\mu \nu}}, \\
\mathcal{F}_{\mu \nu}^{M}= & 2 \partial_{[\mu} A_{\nu]}^{M}-\left[A_{\mu}, A_{\nu}\right]_{E}^{M}+Y_{K L}^{M N} \partial_{N} B_{\mu \nu}^{K L}, \\
\mathcal{F}_{\mu \nu \rho}{ }^{K L}= & 3 \mathcal{D}_{[\mu} B_{\nu \rho]} K L+\frac{3}{D\left(1-2 \beta_{d}\right)} Y_{P Q}^{K L}\left(A_{[\mu}^{(P} \partial_{\nu} A_{\rho]}^{Q)}-\frac{1}{3}\left[A_{[\mu}, A_{\nu}\right]_{E}\left(P A_{\rho]}{ }^{Q)}\right)\right. \\
& -3\left(\partial_{N} C_{\mu \nu \rho}{ }^{N, K L}-Y_{P Q}^{K L} \partial_{N} C_{\mu \nu \rho}{ }^{Q, P N}\right), \\
\mathcal{F}_{\mu \nu \rho \sigma}{ }^{M, K L}= & 4 \mathcal{D}_{[\mu} C_{\nu \rho \sigma]}^{M, K L}+\left(2 B_{[\mu \nu}{ }^{M L} \mathcal{F}_{\rho \sigma]}^{M}-B_{[\mu \nu}{ }^{K L} Y_{P Q}^{M N} \partial_{N} B_{\rho \sigma]}{ }^{P Q}\right) \\
& +\frac{4}{3 D\left(1-2 \beta_{d}\right)} Y_{P Q}^{K L}\left(A_{[\mu}^{M} A_{\nu}^{P} \partial_{\rho} A_{\sigma]}^{Q}-\frac{1}{4} A_{[\mu}^{M}\left[A_{\nu}, A_{\rho}\right]_{E}^{P} A_{\sigma]}^{Q}\right)
\end{aligned}
$$

The higher rank field strengths are related to the lower rank field strengths via the following Bianchi identities

$$
\begin{aligned}
3 \mathcal{D}_{[\mu} \mathcal{F}_{\nu \rho]}^{M} & =-Y_{K L}^{M N} \partial_{N} \mathcal{F}_{\mu \nu \rho}{ }^{K L}, \\
4 \mathcal{D}_{[\mu} \mathcal{F}_{\nu \rho \sigma]}^{K L} & =\frac{1}{2} Y_{P Q}^{K L} \mathcal{F}_{[\mu \nu}{ }^{P} \mathcal{F}_{\rho \sigma]}{ }^{Q}-3\left(\partial_{N} \mathcal{F}_{\mu \nu \rho \sigma}{ }^{N, K L}-Y_{P Q}^{K L} \partial_{N} \mathcal{F}_{\mu \nu \rho \sigma}{ }^{Q, P N}\right), \\
5 \mathcal{D}_{\mu} \mathcal{F}_{\nu \rho \sigma \kappa}^{N, K L} & =\frac{10}{3} \mathcal{F}_{[\mu \nu}^{N} \mathcal{F}_{\rho \sigma \kappa]}{ }^{K L}+\ldots,
\end{aligned}
$$

where the dots in the last line denote terms that always drop from covariant expressions below because of the section condition. 
Under arbitrary variations of the $p$-form potentials the covariant field strengths transform as follows:

$$
\begin{aligned}
\delta \mathcal{F}_{\mu \nu}{ }^{M}= & 2 \mathcal{D}_{[\mu} \Delta A_{\nu]}^{M}-Y_{K L}^{M N} \partial_{N} \Delta B_{\mu \nu}{ }^{K L} \\
\delta \mathcal{F}_{\mu \nu \rho}{ }^{K L}= & 3 \mathcal{D}_{[\mu} \Delta B_{\nu \rho]}{ }^{K L}+\frac{1}{2} Y_{P Q}^{K L} \mathcal{F}_{[\mu \nu}{ }^{P} \Delta A_{\rho]}^{Q} \\
& -3\left(\partial_{N} \Delta C_{\mu \nu \rho}{ }^{N, K L}-Y_{P Q}^{K L} \partial_{N} \Delta C_{\mu \nu \rho}{ }^{Q, P N}\right), \\
\delta \mathcal{F}_{\mu \nu \rho \sigma}{ }^{M, K L}= & 4 D_{[\mu} \Delta C_{\nu \rho \sigma]}^{M, K L}+\frac{1}{18}\left(\frac{3}{8} \mathcal{F}_{[\mu \nu}{ }^{M} \Delta B_{\rho \sigma]}{ }^{K L}-\frac{1}{4} \mathcal{F}_{[\mu \nu \rho} K L \delta A_{\sigma]}^{M}\right)
\end{aligned}
$$

where it proves useful to define "covariant" transformations

$$
\begin{aligned}
\Delta A_{\mu}^{M} & =\delta A_{\mu}^{M}, \\
\Delta B_{\mu \nu}{ }^{K L} & =\delta B_{\mu \nu}{ }^{K L}-\frac{1}{6} Y_{M N}^{K L} A_{[\mu}^{M} \delta A_{\nu]}^{N}, \\
\Delta C_{\mu \nu \rho}{ }^{N, K L} & =\delta C_{\mu \nu \rho}{ }^{N, K L}-\delta A_{[\mu}^{N} B_{\nu \rho]}^{K L}-\frac{1}{18} Y_{R S}^{K L} A_{[\mu}^{N} A_{\nu}^{R} \delta A_{\rho]}^{S} .
\end{aligned}
$$

For the gauge transformations this gives

$$
\begin{aligned}
& \Delta A_{\mu}^{M}=\mathcal{D}_{\mu} \Lambda^{M}+Y_{K L}^{M N} \partial_{N} \Xi_{\mu}{ }^{K L}, \\
& \Delta B_{\mu \nu}{ }^{K L}=2 \mathcal{D}_{[\mu} \Xi_{\nu]}{ }^{K L}-\frac{1}{6} Y_{M N}^{K L} \Lambda^{M} \mathcal{F}_{\mu \nu}{ }^{N}+3\left(\partial_{N} \Psi_{\mu \nu}{ }^{N, K L}-Y_{P Q}^{K L} \partial_{N} \Psi_{\mu \nu}{ }^{P, N Q}\right), \\
& \Delta C_{\mu \nu \rho}{ }^{M, K L}=3 \mathcal{D}_{[\mu} \Psi_{\nu \rho]}{ }^{M, K L}-\mathcal{F}_{[\mu \nu}{ }^{M} \Xi_{\rho]}{ }^{K L}+\frac{1}{9} Y_{P Q}^{K L} \Lambda^{P} \mathcal{F}_{\mu \nu \rho} Q M .
\end{aligned}
$$

The above transformations are constructed such that the covariant 2-, 3- and 4-form field strengths are indeed covariant with respect to $\Lambda^{M}, \Xi_{\mu}{ }^{K L}$ and $\Psi_{\mu \nu}{ }^{N, K L}$ transformations.

Since the 2 - and 3 -forms above are related to the ones parameterizing the supergravity degrees of freedom and duals used in [57] as

$$
\begin{aligned}
B_{\mu \nu i} & =2 \epsilon_{i k l m n} B_{\mu \nu}{ }^{k l m n}, \\
C_{\mu \nu \rho}{ }^{m} & =-6 \epsilon_{n k l r s} C_{\mu \nu \rho}^{m n, k l r s},
\end{aligned}
$$

it is convenient to rewrite the covariant transformation as

$$
\begin{aligned}
\Delta A_{\mu}^{m n} & =\delta A_{\mu}^{m n}, \\
\Delta B_{\mu \nu i} & =\delta B_{\mu \nu i}-2 \epsilon_{i m n k l} A_{[\mu}^{m n} \delta A_{\nu]}^{k l}, \\
\Delta C_{\mu \nu \rho}{ }^{m} & =\delta C_{\mu \nu \rho}^{m}+3 \delta A_{[\mu}^{m n} B_{\nu \rho] n}-2 \epsilon_{n k l r s} A_{[\mu}^{m n} A_{\nu}^{k l} \delta A_{\rho]}^{r s} .
\end{aligned}
$$

Here one should take into account the factor $1 / 2$, that is necessary to prevent double counting when going from capital Latin indices to double small indices in a contraction. With the fields defined in (3.18) and the corresponding relation for the gauge parameters the gauge transformations read

$$
\begin{aligned}
\Delta A_{\mu}^{m n} & =\mathcal{D}_{\mu} \Lambda^{m n}+\frac{1}{16} \epsilon^{i m n k l} \partial_{k l} \Xi_{\mu i}, \\
\Delta B_{\mu \nu i} & =2 \mathcal{D}_{[\mu} \Xi_{\nu] i}-2 \epsilon_{i m n p q} \Lambda^{m n} \mathcal{F}_{\mu \nu}{ }^{p q}-\partial_{m i} \Psi_{\mu \nu}{ }^{m}, \\
\Delta C_{\mu \nu \rho}^{m} & =3 \mathcal{D}_{[\mu} \Psi_{\nu \rho]}^{m}+3 \mathcal{F}_{[\mu \nu}^{m n} \Xi_{\rho] n}+\Lambda^{m n} \mathcal{F}_{\mu \nu \rho n},
\end{aligned}
$$


where the identity (C.5) has been used. Note that these have precisely the same form is in the $D=7$ maximal gauged supergravity up to the following mnemonic replacements of derivatives along extended coordinates by components $Y_{m n}$ and $Z^{m n, k}$ of embedding tensor.

$$
\begin{aligned}
\epsilon^{i m n k l} \partial_{k l} V_{i} & =-16 g Z^{m n, i} V_{i} \\
\partial_{m n} V^{m} & =\frac{g}{24} Y_{m n} V^{m} .
\end{aligned}
$$

Certainly, the correct way to check that the transformations indeed match is to perform Scherk-Schwarz reduction explicitly, possibly, dropping the trombone gauging. Although being an interesting project by itself, this is beyond the scope of the present work.

The same is true for the Bianchi identities that for the fields (3.18) take the following nice form

$$
\begin{aligned}
3 \mathcal{D}_{[\mu} \mathcal{F}_{\nu \rho]}{ }^{m n} & =-\frac{1}{16} \epsilon^{i m n k l} \partial_{k l} \mathcal{F}_{\mu \nu \rho i}, \\
4 \mathcal{D}_{[\mu} \mathcal{F}_{\nu \rho \sigma m} & =6 \epsilon_{m p q r s} \mathcal{F}_{[\mu \nu}{ }^{p q} \mathcal{F}_{\rho \sigma]}{ }^{r s}+\partial_{n m} \mathcal{F}_{\mu \nu \rho \sigma}{ }^{n}, \\
5 \mathcal{D}_{[\mu} \mathcal{F}_{\nu \rho \sigma \kappa]}^{m} & =-10 \mathcal{F}_{[\mu \nu}{ }^{m n} \mathcal{F}_{\rho \sigma \kappa n}+\ldots
\end{aligned}
$$

In the non-coordinate notation the above equations read

$$
\begin{aligned}
\mathcal{D} \mathcal{F}^{m n} & =\frac{1}{16} \epsilon^{i m n k l} \partial_{k l} \mathcal{F}_{i}, \\
\mathcal{D} \mathcal{F}_{m} & =\epsilon_{m p q r s} \mathcal{F}^{p q} \wedge \mathcal{F}^{r s}+\partial_{n m} \mathcal{F}^{n}, \\
\mathcal{D} \mathcal{F}^{m} & =-\frac{1}{5} \mathcal{F}^{m n} \wedge \mathcal{F}_{n}+\ldots,
\end{aligned}
$$

where we define a $p$-form $\omega_{p}$ in terms of its components in the usual way

$$
\omega_{p}=\frac{1}{p !} \omega_{i_{1} \ldots i_{p}} d x^{i_{1}} \wedge \cdots \wedge d x^{i_{p}} .
$$

\section{Covariant exceptional field theory}

The full SL(5)-covariant Exceptional Field Theory Lagrangian has the following structure

$$
\begin{aligned}
\mathcal{L}_{E F T}= & \mathcal{L}_{E H}(\hat{R})+\mathcal{L}_{s c}\left(\mathcal{D}_{\mu} m_{k l}\right)+\mathcal{L}_{V}\left(\mathcal{F}_{\mu \nu}{ }^{m n}\right)+\mathcal{L}_{T}\left(\mathcal{F}_{\mu \nu \rho m}\right) \\
& +\mathcal{L}_{\text {top }}-V\left(m_{k l}, g_{\mu \nu}\right) .
\end{aligned}
$$

Here the modified Einstein-Hilbert term $\mathcal{L}_{E H}$ written in terms of the modified curvature, the kinetic terms for the scalar fields $\mathcal{L}_{s c}$ and for the vectors $\mathcal{L}_{V}$ and the invariant potential for the scalar fields $V$ have the same structural form as in other EFT's, see for example [42, $43,46]$ for the modified EH term and [47] for the general form of the potential.

The kinetic term for the 2 -form gauge potentials $B_{\mu \nu n}$ appears here as a proper Lagrangian governing dynamics of the corresponding degrees of freedom. Whereas, in the $\mathrm{SO}(5,5)$ theory such a term was subject to a self-duality condition and for higher rank U-duality groups was not there at all.

Finally, the topological term has always different structure depending on the dimension and the duality group and hence has to be processed separately. While U-duality covariance 
of the other terms is explicit, the topological term does not have a covariant form. Instead, one may write its variation in a covariant form, that is the only relevant expression to recover EOM's.

We should mention here, that 6+1-dimensional diffeomorphisms for the scalar and vector kinetic terms, the modified Einstein-Hilbert term and the scalar potential work precisely as in $[46,47]$ and hence invariance is not explicitly checked here. However, we perform explicit check of invariance of of the 2-form kinetic term and the topological term with respect to external diffeomorphisms, that successfully fixes all the coefficients in the Lagrangian. This is a known feature of EFT in contrast to the maximal gauged supergravity, where all the coefficients become fixed only after imposing supersymmetry condition. One may speculate that already the bosonic EFT contains some information about the full supersymmetric theory.

\subsection{Kinetic Lagrangian and invariant potential}

The fully covariant Einstein-Hilbert term takes the following usual form

$$
S_{E H}=-\frac{1}{2} \int d^{n} x d^{D} \mathbb{X} e \hat{R}=-\frac{1}{2} \int d^{n} x d^{D} \mathbb{X} e e_{a}^{\mu} e_{b}^{\nu} \hat{R}_{\mu \nu}{ }^{a b},
$$

where the modified curvature reads

$$
\hat{R}_{\mu \nu a b}=R_{\mu \nu a b}+\mathcal{F}_{\mu \nu}^{M} e_{a}^{\rho} \partial_{M} e_{\rho b}
$$

To ensure invariance of the Einstein-Hilbert term with respect to local Lorentz transformations depending on extended coordinates, the corresponding spin-connection $\omega_{\mu}^{a b}$ is set to have weight zero. One should consider this general dependence of local transformations since all fields in the theory depend on the extended coordinates.

The corresponding Lorentz-invariant Riemann scalar then differs from the usual expression and has the same form as in [46]. The usual equation that determines the spinconnection can be written in the following covariant form

$$
\mathcal{D}_{[\mu} e_{\nu]}^{a}-\frac{1}{4} \omega_{[\mu}^{a b} e_{\nu] b}=0 .
$$

As was checked in [47] in general form invariance of the scalar potential implies, that the external vielbein is a generalized scalar of weight $\lambda\left(e_{\mu}^{a}\right)=1 / 5$.

For the scalar degrees of freedom parametrized by the matrix $\mathcal{M}_{M N}$ we just use the general result and set $\alpha_{d}=3[47]$

$$
\begin{aligned}
\mathcal{L}_{s c} & =\frac{1}{12} e g^{\mu \nu} \mathcal{D}_{\mu} \mathcal{M}_{M N} \mathcal{D}_{\nu} \mathcal{M}^{M N}, \\
\mathcal{M}_{m n k l} & =m_{m k} m_{n l}-m_{m l} m_{n k}
\end{aligned}
$$

As expected this is explicitly covariant under the local gauge transformation generated by the generalized Lie derivative. 
Finally, the kinetic terms for the 1 -form potential $A_{\mu}^{M}$ and the 2 -form potential $B_{\mu \nu m}$ take the following form

$$
\begin{aligned}
\mathcal{L}_{V} & =-\frac{1}{4} e \mathcal{M}_{M N} \mathcal{F}_{\mu \nu}{ }^{M} \mathcal{F}_{\mu \nu}{ }^{N}=-\frac{1}{8} e m_{m k} m_{n l} \mathcal{F}_{\mu \nu}{ }^{m n} \mathcal{F}^{\mu \nu k l}, \\
\mathcal{L}_{T} & =-\frac{1}{3 \cdot(16)^{2}} e m^{m n} \mathcal{F}_{\mu \nu \rho m} \mathcal{F}^{\mu \nu \rho}{ }_{n}
\end{aligned}
$$

Each term here is separately covariant with respect to generalized diffeomorphisms and all gauge transformations. It is explicitly shown further, that the numerical coefficient in $\mathcal{L}_{T}$ is defined by invariance under 6+1-dimensional external diffeomorphisms.

The non-topological part of the Lagrangian is concluded by the so-called scalar potential. This depends only on the scalar and metric degrees of freedom and their derivatives with respect to extended coordinates $\mathbb{X}^{m n}$. This has a form universal for the duality groups $E_{6,5}, \mathrm{SO}(5,5), \mathrm{SL}(5)$ and $\mathrm{SL}(2) \times \mathrm{SL}(3)$ :

$$
\begin{aligned}
V= & -\frac{1}{4 \alpha_{d}} \mathcal{M}^{M N} \partial_{M} \mathcal{M}^{K L} \partial_{N} \mathcal{M}_{K L}+\frac{1}{2} \mathcal{M}^{M N} \partial_{M} \mathcal{M}^{K L} \partial_{L} \mathcal{M}_{N K} \\
& -\frac{1}{2}\left(g^{-1} \partial_{M} g\right) \partial_{N} \mathcal{M}^{M N}-\frac{1}{4} \mathcal{M}^{M N}\left(g^{-1} \partial_{M} g\right)\left(g^{-1} \partial_{N} g\right)-\frac{1}{4} \mathcal{M}^{M N} \partial_{M} g^{\mu \nu} \partial_{N} g_{\mu \nu},
\end{aligned}
$$

where the terms in the first line are precisely those of [5] and the rest terms are needed to ensure gauge invariance, and one should note the determinant $\sqrt{-g}$ in the action. For the case in question one sets $\alpha_{d}=3$. Covariance of the above expression has been explicitly checked in [47].

\subsection{Topological Lagrangian}

To construct the topological term one notes that in the embedding tensor formulation of the maximal $D=7$ supergravity the field $C_{\mu \nu \rho}{ }^{m}$ appears only under projection with the tensor

$$
Y_{m n}=\frac{1}{2} \Theta_{p(m, n)}^{p},
$$

that parametrizes gaugings in the $\mathbf{1 5}$ of SL(5). Analyzing the expressions for the 3 -form field strength $\mathcal{F}_{\mu \nu \rho}{ }^{K L}$ and for the covariant transformation $\Delta B_{\mu \nu}{ }^{K L}$ one arrives to the following rule

$$
Y_{m n} C_{\mu \nu \rho}{ }^{n} \rightarrow \epsilon_{m K L}\left(\partial_{N} C_{\mu \nu \rho}^{N, K L}-Y_{P Q}^{K L} \partial_{N} C_{\mu \nu \rho}^{Q, P N}\right) .
$$

Indeed, since the field $C_{\mu \nu \rho}{ }^{m n, k l r s}$ contains only the representation $\mathbf{5}$ of $\mathrm{SL}(5)$ one has the identity $12 \epsilon_{p k l r s} C_{\mu \nu \rho}{ }^{m n, k l r s}=C_{\mu \nu \rho}{ }^{[m} \delta_{p}^{n]}$. Now performing Scherk-Schwarz reduction $C_{\mu \nu \rho}^{r}\left(x^{\mu}, \mathbb{X}^{M}\right)=V_{\bar{r}}^{r}\left(\mathbb{X}^{M}\right) C^{\bar{r}}\left(x^{\mu}\right)$ one obtains

$$
\epsilon_{i K L}\left(\partial_{N} C_{\mu \nu \rho}^{N, K L}-Y_{P Q}^{K L} \partial_{N} C_{\mu \nu \rho}^{Q, P N}\right) \rightarrow\left(V_{\bar{p}}^{p} \partial_{p q} V_{\bar{q}}^{q}\right) C_{\mu \nu \rho} \bar{q} .
$$

Using the SL(5) gaugings written in terms of twist matrices obtained in [18] the expression in brackets on the r.h.s. becomes precisely $Y_{\bar{p} \bar{q}}$ (for the vanishing trombone gauging and $\operatorname{det} V=1)$. Barred indices are used only in the paragraph above and denote the flat directions of the Scherk-Schwarz twist matrix, see [18, 19]. 
Hence, inspired by these observations, we write variation of the topological Lagrangian in the following simple form (cf. [57])

$$
\begin{aligned}
\delta \mathcal{L}_{\text {top }}=A \epsilon^{\mu \nu \rho \lambda \sigma \tau \kappa} & {\left[\mathcal{F}_{\mu \nu \rho \lambda}{ }^{i} \epsilon_{i K L}\left(\partial_{N} \Delta C_{\sigma \tau \kappa}^{N, K L}-Y_{P Q}^{K L} \partial_{N} \Delta C_{\sigma \tau \kappa}^{Q, P N}\right)\right.} \\
& \left.+\frac{1}{4} \mathcal{F}_{\mu \nu}{ }^{i j} \mathcal{F}_{\rho \lambda \sigma i} \Delta B_{\tau \kappa j}-\frac{1}{12} \mathcal{F}_{\mu \nu \rho i} \mathcal{F}_{\lambda \sigma \tau j} \delta A_{\kappa}^{i j}\right]+ \text { total derivatives. }
\end{aligned}
$$

Here the coefficients are chosen for the variation to vanish on all gauge and U-duality transformation. Since it is easier to work with the fields (3.18) we rewrite the above expression as

$$
\delta \mathcal{L}_{\text {top }}=A \epsilon^{\mu \nu \rho \lambda \sigma \tau \kappa}\left[\mathcal{F}_{\mu \nu \rho \lambda}{ }^{i} \partial_{i j} \Delta C_{\sigma \tau \kappa}^{j}+6 \mathcal{F}_{\mu \nu}^{i j} \mathcal{F}_{\rho \lambda \sigma i} \Delta B_{\tau \kappa j}-2 \mathcal{F}_{\mu \nu \rho i} \mathcal{F}_{\lambda \sigma \tau j} \delta A_{\kappa}^{i j}\right],
$$

where the overall prefactor will be fixed to $A^{-1}=16 \cdot 4$ ! by invariance with respect to $6+1$-dimensional external diffeomorphisms. Note, that $\epsilon^{\mu \nu \rho \sigma \lambda \tau \kappa}$ here and always in the paper is the alternating symbol, rather than the Levi-Civita tensor, and hence does not contain the determinant $e$.

As for the topological terms of EFT's in other dimensions (as well as of gauged supergravities) this expression cannot be written as variation of a covariant expression. However, the above is enough to write equations of motion and to check invariance of the Lagrangian with respect to $6+1$-dimensional diffeomorphisms.

\section{3 $D=6+1$ diffeomorphisms}

In the previous section we have established the explicit form of the kinetic Lagrangian for the fields $A_{\mu}{ }^{m n}, B_{\mu \nu m}$ and $\mathcal{M}^{M N}$, the modified Einstein-Hilbert term, the scalar potential $V(\partial \mathcal{M}, \mathcal{M})$ and the topological term. These are invariant under duality transformations as well as under all the gauge transformations resulting from the tensor hierarchy. This invariance fixed for us all the mutual prefactors in the Lagrangian except the prefactor of the topological term and the kinetic term of the 2 -form gauge potential. It is known, that the same situation appears in the maximal gauged supergravity models, where to fix the remaining prefactor on needs to consider supersymmetry.

The case of Exceptional Field Theory is different due to dependence of all the fields on the extra coordinates $\mathbb{X}^{M}$. This results in the fact, that external $6+1$-dimensional diffeomorphisms do not work automatically and one has to perform a certain check of that. Remarkably, it is enough just to fix these two prefactors to satisfy the invariance condition. The result is a completely fixed duality, gauge and external diffeomorphism invariant Lagrangian. Another miracle appears, when one checks supersymmetry of the (SUSY extended) Lagrangian and gets that for free. This has been checked explicitly for the duality groups $E_{7,6}$ in [49] and [50], however there is no reason to expect, that other U-duality groups fail to follow this scheme. 
Hence, let us start with the following external diffeomorphism transformations

$$
\begin{aligned}
\delta e_{\mu}^{a} & =\xi^{\mu} \mathcal{D}_{\nu} e_{\mu}^{a}+\mathcal{D}_{\mu} \xi^{\nu} e_{\nu}^{a} \\
\delta \mathcal{M}_{M N} & =\xi^{\mu} \mathcal{D}_{\mu} \mathcal{M}_{M N} \\
\delta A_{\mu}{ }^{M} & =\xi^{\nu} \mathcal{F}_{\nu \mu}{ }^{M}+\mathcal{M}^{M N} g_{\mu \nu} \partial_{N} \xi^{\nu} \\
\Delta B_{\mu \nu i} & =\xi^{\rho} \mathcal{F}_{\rho \mu \nu i}, \\
\Delta C_{\mu \nu \rho}{ }^{m} & =-\frac{1}{3 !} e \epsilon_{\mu \nu \rho \sigma \kappa \lambda \tau} \xi^{\sigma} m^{m n} \mathcal{F}^{\kappa \lambda \tau}{ }_{n},
\end{aligned}
$$

where $\varepsilon_{\mu \nu \rho \sigma \kappa \lambda \tau}=e \epsilon_{\mu \nu \rho \sigma \kappa \lambda \tau}$ is the Levi-Civita tensor in 7 dimensions. Transformation of the 3 -form potential is required to be of this particular form by off-shell invariance of the Lagrangian (see remark at the end of this section).

One should note, that huge part of cancellations here works precisely as in the maximal gauged supergravity and hence, does not need to be double checked. In contrast, the terms that contain the derivative $\partial_{m n} \xi^{\mu}$ do not exist in the gauged models and hence need to be processed explicitly. We will refer to them as new terms and work in the close analogy to [46]. Next we note that diffeomorphism invariance of the universal scalar potential $V$ has been checked in general form in [47], hence we just use the result here.

Let us start with variations of the 2 - and 3 -form field strengths and write for the former

$$
\begin{aligned}
\delta \mathcal{F}_{\mu \nu}{ }^{m n} & =2 \mathcal{D}_{[\mu} \Delta A_{\nu]}^{m n}-\frac{1}{16} \epsilon^{m n p q r} \partial_{p q} B_{\mu \nu r} \\
& =2 \mathcal{D}_{[\mu}\left(\xi^{\rho} \mathcal{F}_{\rho \mu}^{m n}\right)+\mathcal{D}_{[\mu}\left(\mathcal{M}^{m n, p q} g_{\nu] \rho} \partial_{p q} \xi^{\rho}\right)-\frac{1}{16} \epsilon^{m n p q r} \partial_{p q} \partial_{p q}\left(\xi^{\rho} \mathcal{F}_{\mu \nu \rho r}\right) \\
& =\left(L_{\xi}^{\mathcal{D}} \mathcal{F}_{\mu \nu}{ }^{m n}\right)-\frac{1}{16} \epsilon^{m n p q r} \mathcal{F}_{\mu \nu \rho r} \partial_{p q} \xi^{\rho}+2 \mathcal{D}_{[\mu}\left(m^{m p} m^{n q} g_{\nu] \rho} \partial_{p q} \xi^{\rho}\right)
\end{aligned}
$$

where we used the Bianchi identity for the field $\mathcal{F}_{\mu \nu}{ }^{m n}$ to organize the (conventional) Lie derivative with respect to $\mathcal{D}_{\mu}$ that is denoted by $L_{\xi}^{\mathcal{D}}$. Using the same arguments and the Bianchi identity for the 3 -form field strength we write for its variation

$$
\begin{aligned}
\delta \mathcal{F}_{\mu \nu \rho m}= & 3 \mathcal{D}_{[\mu} \Delta B_{\nu \rho] m}+6 \epsilon_{m p q r s} \mathcal{F}_{[\mu \nu}^{p q} \delta A_{\rho]}{ }^{r s}-\partial_{m n} \Delta C_{\mu \nu \rho}{ }^{n} \\
= & 3 \mathcal{D}_{[\mu}\left(\xi^{\sigma} \mathcal{F}_{\nu \rho \sigma] m}\right)+6 \epsilon_{m p q r s} \mathcal{F}_{[\mu \nu}^{p q} \mathcal{F}_{\sigma \rho]}{ }^{r s} \xi^{\sigma}+3 \epsilon_{m p q r s} \mathcal{F}_{[\mu \nu}{ }^{p q} \mathcal{M}^{r s, k l} g_{\rho \sigma} \partial_{k l} \xi^{\sigma} \\
& -\partial_{m n} \Delta C_{\mu \nu \rho}{ }^{n} \\
= & \left(L_{\xi}^{\mathcal{D}} \mathcal{F}_{\mu \nu \rho m}\right)+6 \epsilon_{m p q r s} m^{r k} m^{s l} \mathcal{F}_{\mu \nu}{ }^{p q} g_{\rho \sigma} \partial_{k l} \xi^{\sigma}+\xi^{\sigma} \partial_{m n} \mathcal{F}_{\mu \nu \rho \sigma}{ }^{n}-\partial_{m n} \Delta C_{\mu \nu \rho}{ }^{n} .
\end{aligned}
$$

Here the transformation of the 3 -form gauge potential $\Delta C_{\mu \nu \rho}{ }^{m}$ was left inexplicit for further convenience.

Now, one notices that terms in the variation of the Lagrangian containing the (conventional) Lie derivative of the 2 - and 3 -form potentials together with variations of the determinant of the vielbein det $e$ and the scalar matrix $m^{m n}$ in the corresponding kinetic terms give just full derivative. This is exactly the same as in the gauged theories and in other EFT's. Next, from the analysis of EFT's for the other U-duality groups one concludes that the last term in the last line of (4.14) will cancel against the corresponding contribution from the variation of the modified Einstein-Hilbert term. 
Hence, what is left are the following six terms

$$
\begin{aligned}
& (1)=\frac{1}{4 \cdot 16} e m_{m k} m_{n l} \epsilon^{m n p q r} \mathcal{F}^{\mu \nu k l} \mathcal{F}_{\rho \mu \nu r} \partial_{p q} \xi^{\rho}, \\
& (2)=-\frac{1}{4 \cdot 16} e m^{m n} \epsilon_{m p q r s} m^{r k} m^{s l} \mathcal{F}_{\mu \nu}{ }^{p q} \mathcal{F}^{\mu \nu \rho}{ }_{n} g_{\rho \sigma} \partial_{k l} \xi^{\sigma}, \\
& (3)=\frac{1}{16} e m^{m n} \mathcal{F}^{\mu \nu \rho}{ }_{n}\left(\xi^{\sigma} \partial_{m k} \mathcal{F}_{\mu \nu \rho \sigma}{ }^{k}-\partial_{m k} \Delta C_{\mu \nu \rho}{ }^{k}\right), \\
& (4)=A \epsilon^{\mu \nu \rho \lambda \sigma \tau \kappa} \mathcal{F}_{\mu \nu \rho \lambda}{ }^{m} \partial_{m n} \Delta C_{\sigma \tau \kappa}^{n}, \\
& (5)=-2 A \epsilon^{\mu \nu \rho \lambda \sigma \tau \kappa} \mathcal{F}_{\mu \nu \rho m} \mathcal{F}_{\lambda \sigma \tau n}\left(\xi^{\psi} \mathcal{F}_{\psi \kappa}{ }^{m n}+g_{\kappa \psi} m^{m k} m^{n l} \partial_{k l} \xi^{\psi}\right), \\
& (6)=6 A \epsilon^{\mu \nu \rho \lambda \sigma \tau \kappa} \mathcal{F}_{\mu \nu}{ }^{m n} \mathcal{F}_{\rho \lambda \sigma m} \mathcal{F}_{\psi \tau \kappa n} \xi^{\psi}
\end{aligned}
$$

where the first line comes from the variation of the kinetic term for the 1-form gauge potential, the second and third lines comes from the kinetic term for the 2-form gauge potential. The last three lines result from the expression (4.12), with the line (5) resulting from the term with $\delta A_{\mu}^{m n}$ and the line (6) from the term with $\Delta B_{\mu \nu m}$. One immediately notes here, that the first term in brackets in the line (5) together with the line (6) forms an expression with the 8 indices $\{\mu \nu \rho \lambda \sigma \tau \kappa \psi\}$ fully antisymmetrized, and hence cancels.

Next, the lines (1) and (2) cancel against each other since the scalar matrix $m \in \mathrm{SL}(5)$. Indeed, as a consequence of $\operatorname{det} m=1$ we may write

$$
m^{m n} m^{r k} m^{s l} \epsilon_{m p q r s}=\epsilon^{n k l i j} m_{i p} m_{j q}
$$

that after substituting into (2) gives the desired cancellation. Note, that the identity above can be understood as a rule for raising and lowering the indices of the alternating symbol, however we will not need this.

Now, we note that the first term [3.1] in the line (3) and the term in the line (4) can be combined into a full derivative. Indeed, using the transformation of the 3 -form gauge potential we write

$$
\begin{aligned}
& {[3.1]+(4)=} \\
& =-\frac{1}{16} e m^{n k} \mathcal{F}^{\mu \nu \rho}{ }_{k} \xi^{\sigma} \partial_{n m} \mathcal{F}_{\mu \nu \rho \sigma}{ }^{m}-\frac{A}{3 !} \epsilon^{\mu \nu \rho \lambda \sigma \tau \kappa} \mathcal{F}_{\mu \nu \rho \lambda}{ }^{m} \partial_{m n}\left(e \epsilon_{\sigma \tau \kappa \psi \chi} \tau \omega \xi^{\psi} \mathcal{F}^{\chi \tau \omega}{ }_{k} m^{n k}\right) \\
& =-\frac{1}{16} e m^{n k} \mathcal{F}^{\mu \nu \rho}{ }_{k} \xi^{\sigma} \partial_{n m} \mathcal{F}_{\mu \nu \rho \sigma}{ }^{m}-A \cdot 4 ! \mathcal{F}_{\mu \nu \rho \sigma}{ }^{m} \partial_{m n}\left(e \xi^{\mu} \mathcal{F}^{\nu \rho \sigma}{ }_{k} m^{n k}\right) .
\end{aligned}
$$

Setting the prefactor $A^{-1}=16 \cdot 4$ ! and taking into account two minus signs resulting from necessary permutation, one arrives to a full derivative, that drops from the variation of the action.

With the prefactor $A$ being fixed we are left with check of the cancellation between the second terms in the lines (3) and (5). This is straightforward, however one should take care of the det $e$ prefactors. Hence, we write (multiplied by $(32 \cdot 3$ !) for convenience)

$$
\begin{aligned}
& (32 \cdot 3 !)([3.2]+[5.2])= \\
& =-2 e m^{m n} \mathcal{F}^{\mu \nu \rho}{ }_{n} \partial_{m k}\left(e \epsilon_{\mu \nu \rho \sigma \kappa \lambda \tau} \xi^{\sigma} \mathcal{F}^{\kappa \lambda \tau}{ }_{l} m^{k l}\right)-\epsilon^{\mu \nu \rho \lambda \sigma \tau \kappa} \mathcal{F}_{\mu \nu \rho m} \mathcal{F}_{\lambda \sigma \tau n} g_{\kappa \psi} m^{m k} m^{n l} \partial_{k l} \xi^{\psi}
\end{aligned}
$$




$$
\begin{aligned}
& =-2 \epsilon_{\mu \nu \rho \sigma \kappa \lambda \tau} \partial_{m k}\left(e m^{m n} \mathcal{F}^{\mu \nu \rho}{ }_{n}\right) e \mathcal{F}^{\kappa \lambda \tau}{ }_{l} m^{k l} \xi^{\sigma}-e \cdot \varepsilon_{\mu \nu \rho \lambda \sigma \tau \kappa} \mathcal{F}^{\mu \nu \rho}{ }_{m} \mathcal{F}^{\lambda \sigma \tau}{ }_{n} m^{m k} m^{n l} \partial_{k l} \xi^{\kappa} \\
& =-\epsilon_{\mu \nu \rho \sigma \kappa \lambda \tau} \partial_{m k}\left(e^{2} m^{m n} \mathcal{F}^{\mu \nu \rho}{ }_{n} \mathcal{F}^{\kappa \lambda \tau}{ }_{l} m^{k l} \xi^{\sigma}\right) \Rightarrow 0 .
\end{aligned}
$$

Note the use of the Levi-Civita tensor $\varepsilon_{\mu \nu \rho \lambda \sigma \tau \kappa}$ in the second expression, that produces an extra factor of $e$ times the (constant) alternating symbol.

As the final remark in this section let us look at the equations of motion for the (non-dynamical) field $C_{\mu \nu \rho}{ }^{m}$, that read

$$
\partial_{m k}\left(e m^{m n} \mathcal{F}_{n}^{\mu \nu \rho}-\frac{1}{4 !} \epsilon^{\mu \nu \rho \lambda \sigma \tau \kappa} \mathcal{F}_{\lambda \sigma \tau \kappa}{ }^{m}\right)=0
$$

The result is that the 3 -form gauge potential does not give dynamical field equations in the external 6+1-dimensional space-time. Rather, it results in restricting of the 3-and 4-form field strength behavior in the internal extended space. After Scherk-Schwarz reduction the above equation results in the known duality relation between the 3 - and 4-form field strengths. This is an expected result, as the fifth component of the 2-form gauge potential was introduced as a dualization of the 11-dimensional 3-form gauge field with all indices external. Since one was always allowed to dualize the 2 -form gauge degrees of freedom to get a 3-form gauge potential, to keep the story duality covariant one should introduce both the 2 - and the 3 -form gauge potentials. This doubling of fields is the price for having the theory duality covariant. The final field content of the model depends on the gauging chosen.

Finally, let us note, that upon imposing the following dualization constraint

$$
e m^{m n} \mathcal{F}_{n}^{\mu \nu \rho}-\frac{1}{4 !} \epsilon^{\mu \nu \rho \lambda \sigma \tau \kappa} \mathcal{F}_{\lambda \sigma \tau \kappa}{ }^{m},
$$

the diffeomorphism transformation rule for the 3-form field strength takes its conventional form

$$
\Delta_{\xi} C_{\mu \nu \rho}^{m}=\xi^{\sigma} \mathcal{F}_{\sigma \mu \nu \rho}{ }^{m}
$$

\section{Embeddings of $\mathrm{D}=11$ and Type IIB supergravity}

The field content of the 11-dimensional and Type IIB supergravity can be naturally embedded into the field content of the exceptional field theory upon a correct choice of the solution of the section condition. Depending on the duality group one gets a different splitting of the coordinates of the resulting theory. As was shown in [46] for the $\mathrm{E}_{6}$ exceptional field theory the resulting Lagrangian does not preserve the full $\mathrm{D}=10$ Lorenz invariance due to this coordinate split. Since there is no reason to expect that on the level of the Lagrangian the construction works only for the $\mathrm{E}_{6}$ duality group, where it has been checked explicitly, we perform here only the check of the field content. However, in principle one would be interested in having an explicit picture of how the Lagrangian of all the EFT's reduces to the known supergravities.

Let us start with embedding of the 11-dimensional supergravity field content. The corresponding solution of the section condition breaks the U-duality group SL(5) to GL(4) 
and provides the following decomposition of the relevant representations

$$
\begin{aligned}
\mathrm{SL}(5) & \rightarrow \mathrm{SL}(4) \times G L(1) \simeq G L(4) ; \\
\mathbf{1 0} & \rightarrow \mathbf{4}_{-3}+\mathbf{6}_{2} \\
\mathbf{5} & \rightarrow \mathbf{4}_{1}+\mathbf{1}_{-4} \\
\mathbf{2 4} & \rightarrow \mathbf{1}_{0}+\mathbf{4}_{5}+\overline{\mathbf{4}}_{-5}+\mathbf{1 5}_{0}
\end{aligned}
$$

where the subscripts denote weights with respect to the $G L(1)$ subgroup. Since the extended coordinates $\mathbb{X}^{m n}$ transform under the representation 10 they decompose according to the second line above, that gives

$$
\mathbb{X}^{m n} \rightarrow\left\{\mathbb{X}^{5 a}, \mathbb{X}^{a b}\right\} \rightarrow\left\{x^{a}, \epsilon^{a b c d} y_{c d}\right\}
$$

where $\epsilon^{a b c d}$ is the alternating symbol in 4 dimensions. The coordinates $x^{a}$ have the interpretation of the usual geometric coordinates, while $y_{a b}$ correspond to winding modes of the M2-brane. It is straightforward to check that dropping dependence on the winding coordinates solves the section condition

$$
\epsilon^{i m n k l} \partial_{m n} \otimes \partial_{k l}=0
$$

Hence, all the fields of the theory depend only on eleven coordinates: the space-time external coordinates $x^{\mu}$ and the internal ones $x^{a}$.

The corresponding decomposition of the gauge fields works as follows

$$
\begin{aligned}
& A_{\mu}^{m n} \rightarrow A_{\mu}^{a}, A_{\mu a b}, \\
& B_{\mu \nu m} \rightarrow B_{\mu \nu}, B_{\mu \nu a} .
\end{aligned}
$$

Here we do not include the field $C_{\mu \nu \rho}{ }^{m}$ as it completely drops from the theory on the solution of the section condition. This decomposition nicely fits into the decomposition of the metric $G_{\widehat{M} \hat{N}}$ and the 3 -form field $C_{\widehat{\mathbb{N} \hat{N}}}$ in 11 dimensions (see (2.1))

$$
\begin{aligned}
G_{\hat{\mathbb{N}} \hat{N}} & \rightarrow\left\{g_{\mu \nu}, A_{\mu}^{a}, \varphi_{a b}\right\}, \\
C_{\hat{\mathbb{N} N \hat{K}}} & \rightarrow\left\{C_{\mu \nu \rho}, B_{\mu \nu a}, A_{\mu a b}, \varphi_{a b c}\right\}
\end{aligned}
$$

The 3-form field $C_{\mu \nu \rho}$ is obtained by dualization of the 2-form $B_{\mu \nu}$ in 7 dimensions. The 14 scalars $\varphi_{a b}$ and $\varphi_{a b c}$ is the above decomposition are identified with the components of the generalized metric $m_{m n}$ that lives in the $\mathbf{2 4}$ of SL(5) factorized by the $\mathbf{1 0}$ of $\mathrm{SO}(5)$ considered as its subgroup. Hence a combination of $\mathbf{4}_{\mathbf{5}}$ and $\overline{\mathbf{4}}_{-\mathbf{5}}$ is factored out as well as the $\mathrm{SO}(4)$ part of the $\mathbf{1 5}_{\mathbf{0}}$. The latter together with the singlet $\mathbf{1}$ form the coset space $\mathrm{GL}(4) / \mathrm{SO}(4)$, while the remained 4 gives the fields $\varphi_{a b c}$. The easiest way to see this is to look at decomposition in the matrix representation of the groups $\mathrm{SL}(5)$ and $\mathrm{SO}(5)$

$$
\mathrm{SL}(5):\left[\begin{array}{cc}
\mathrm{SL}(4) & \mathbf{4}_{\mathbf{5}} \\
\overline{4}_{-\mathbf{5}} & \mathbf{1}_{\mathbf{0}}
\end{array}\right], \quad \mathrm{SO}(5):\left[\begin{array}{cc}
\mathrm{SO}(4) & \mathbf{4} \\
\mathbf{4} & 1
\end{array}\right]
$$

The other possible branching $\mathrm{SL}(5) \rightarrow \mathrm{GL}(3) \times \mathrm{SL}(2)$ gives the field content of the tendimensional Type IIB supergravity with $7+3$ split. As usual for EFT's, the explicit SL(2) 
symmetry is identified with the S-duality symmetry of the theory. Branching rules for the relevant representations take the following form

$$
\begin{aligned}
\mathbf{1 0} & \rightarrow(\mathbf{1}, \mathbf{1})_{-6}+(\overline{\mathbf{3}}, \mathbf{1})_{4}+(\mathbf{3}, \mathbf{1})_{-1}, \\
\mathbf{5} & \rightarrow(\mathbf{1}, \mathbf{2})_{-3}+(\mathbf{3}, \mathbf{1})_{2}, \\
\mathbf{2 4} & \rightarrow(\mathbf{1}, \mathbf{1})_{0}+(\mathbf{1}, \mathbf{3})_{0}+(\mathbf{3}, \mathbf{2})_{5}+(\overline{\mathbf{3}}, \mathbf{2})_{-5}+(\mathbf{8}, \mathbf{1})_{0},
\end{aligned}
$$

where the first irrep corresponds to the SL(3) subgroup of GL(3) and the subscript denotes weight with respect to its GL(1) subgroup. The first line above implies the following decomposition of the extended coordinates $\mathbb{X}^{m n}$

$$
\mathbb{X}^{m n} \rightarrow\left\{\mathbb{X}^{\underline{a b}}, \mathbb{X}^{\underline{a} \hat{\alpha}}, \mathbb{X}^{\hat{\hat{\beta}} \hat{\beta}}\right\} \rightarrow\left\{\epsilon^{a b c} x_{\underline{c}}, y^{\underline{a}, \hat{\alpha}}, \epsilon^{\hat{\alpha} \hat{\beta}} z\right\},
$$

where $\epsilon^{a b c}$ and $\epsilon^{\hat{\alpha} \hat{\beta}}$ are the alternating symbols for the SL(3) and SL(2) groups respectively Supergravity interpretation of the above decomposition in terms of the geometric coordinates and winding modes of various Type II branes needs more careful consideration.

First, one should note that breaking the SL(2) symmetry explicitly and leaving only the coordinates $\left\{x_{\underline{a}}, y^{\text {una, } 1}\right\}$ results in the $\mathrm{O}(\mathrm{d}, \mathrm{d})$ theory, that is the Double Field Theory [58]. The DFT section condition

$$
\frac{\partial}{\partial x_{\underline{a}}} \otimes \frac{\partial}{\partial y \underline{a}}=0
$$

is a direct consequence of the $\mathrm{SL}(5)$ section condition. Hence, as it is known from DFT, to return to the Type IIA theory one just drops dependence on the $x_{\underline{a}}$ coordinates, that correspond to winding of the fundamental string of Type IIA. Alternatively, to end up with Type IIB theory one drops the dual coordinates $y^{\underline{a}}$ and interprets what remains as the normal geometric coordinates. In the recent work [22] this procedure was used to obtain consistent truncations of Type IIA and IIB supergravities from the SL(5) extended geometry.

With this in mind we return back to the decomposition (5.8) and identify the $x_{\underline{a}}$ with the geometric coordinates while the doublet $y^{\underline{a} \hat{\alpha}}$ is identified with the doubled of winding modes for the fundamental string and the D1 brane. The latter are indeed dual with respect to an S-duality rotation. Finally the coordinate $z$ is understood as winding mode for the D3 brane in 3 dimensions. It is important to mention that the SL(2) symmetry is not broken.

To identify the fields of the SL(5) EFT let us look at the $7+3$ decomposition of the (bosonic) fields of Type IIB supergravity

$$
\begin{aligned}
& G_{\mathrm{MN}} \longrightarrow g_{\mu \nu}, A_{\mu \underline{a}}, \varphi_{\underline{a b}} ; \\
& C_{\hat{\alpha}} \longrightarrow \varphi_{\hat{\alpha}} ; \\
& B_{\mathrm{MN} \hat{\alpha}} \longrightarrow B_{\mu \nu \hat{\alpha}}, A_{\mu \underline{a} \hat{\alpha}}, \varphi_{\underline{a b} \hat{\alpha}} ; \\
& C_{\mathrm{MNKL}} \longrightarrow B_{\mu \nu \underline{a b}}, A_{\mu}, C_{\mu \nu \rho \sigma}, C_{\mu \nu \rho \underline{a}} .
\end{aligned}
$$

Note that die to the self-duality of the 4-form gauge potential in 10 dimensions only the half of d.o.f. in the last line above survives. The branching rule imply the following decomposition of the EFT gauge fields

$$
\begin{aligned}
& A_{\mu}^{m n} \rightarrow\left\{A_{\mu}, A_{\mu \underline{a}}, A_{\mu} \underline{\underline{a} \hat{\alpha}}\right\}, \\
& B_{\mu \nu m} \rightarrow\left\{B_{\mu \nu \underline{a}}, B_{\mu \nu \hat{\alpha}}\right\} .
\end{aligned}
$$


Considering only the fields $C_{\mu \nu \rho \underline{a}}$ and $A_{\mu}$ of the last line in (5.10) as physical we identify them with the field $A_{\mu}$ of EFT and the dual of $B_{\mu \nu \underline{a}}$. Note that the underlined indices labeling the $\mathbf{3}$ of SL(3) can be raised and lowered by the scalar matrix. With this in hand one directly identifies the remaining gauge fields.

The generalized metric $m_{m n}$ represented by the coset element can be decomposed as follows

$$
m_{m n} \rightarrow\left\{m_{\underline{a b}}, m_{\underline{a} \hat{\alpha}}, m_{\hat{\alpha} \hat{\beta}}\right\} .
$$

Here the fields $m_{\underline{a} \hat{\alpha}}$ are directly identified with those coming from the 2-form in 10 dimensions up to contraction with the alternating symbol $\epsilon \frac{a b c}{}$. The element $m_{\underline{a b}}$ of the coset $\mathrm{GL}(3) / \mathrm{SO}(3)$ give the internal part $\varphi_{\underline{a b}}$ of the 10-dimensional metric, while the 2 fields $m_{\underline{\alpha} \beta}$ parameterizing the coset $\mathrm{SL}(2) / \mathrm{SO}(2)$ match the axion-dilaton $C_{\hat{\alpha}}$. One may come to the same conclusions by analyzing the coset decomposition of the generalized vielbein, however we find the above analysis more transparent.

Hence, we conclude that the expected result of recovering the 11-dimensional supergravity and Type IIB supergravity by different solutions of the section condition holds for the SL(5) theory as for the other EFT's. The same procedure has been used in [20] to explicitly obtain the Lagrangian for Type IIB and 11-dimensional supergravities from the internal sector of EFT, developed in [4] and [5]. One is still interested in doing the same for the full SL(5) EFT and for its supersymmetric extension.

\section{Discussion and outlook}

In this work the construction of SL(5) Exceptional Field Theory was presented, that fills the empty slot in the chain of EFT's for the groups $E_{8,7,6}, \mathrm{SO}(5,5)$ and $\mathrm{SL}(3) \times \mathrm{SL}(2)$ already constructed. These correspond to the maximal supergravities in $D=3,4,5,6$ and $D=8$ respectively. Hence, the presented model adds the $D=7$ case and fulfills the chain. The U-duality groups for $D=9,10$ supergravity are too simple and the extended space can not be constructed. On the other end one meets the $E_{9}$ group to be expected as the U-duality group for $D=2$ maximal supergravity. This is infinitely dimensional, and hence the extended geometry in its known form ruins here as well.

For some applications, such as searches for solutions or classification of gaugings, models with SL(5) U-duality group seem to be more convenient as these provide less extended coordinates and carry more simple algebraic structure.

Although the initial construction of extended geometry has resulted from investigation of the toroidal backgrounds in supergravity, it is in general believed, that Scherk-Schwarz compactifications are able to catch non-toroidal and even non-geometric backgrounds. There was large progress in the direction of uplifting Type IIB solutions and solutions of 11-dimensional supergravity into EFT by choosing an appropriate Scherk-Schwarz reduction scheme [22-24]. However, there is still discussion in the literature, whether one should use other approaches to describe non-toroidal backgrounds. One of them is the so called WZW Double Field Theory, that attempts to construct a DFT on a group manifold [59-62]. hence, in this context it would be interesting to expand the ideas of exceptional field theory to $\mathrm{DFT}_{W Z W}$ and look for possible uplifts. 
The presented theory is essentially bosonic and one may be interested in extending it to include fermions in a supersymmetry invariant way. For the $\mathrm{E}_{6,7}$ EFT's this was done in $[49,50]$. The interesting point here is that in contrast to the maximal gauged theories the bosonic Lagrangian is completely fixed already one the bosonic level with no need of supersymmetry. Hence, the fermionic sector should be constructed in such a way to fit nicely in the existing theory. With such theory in hands one may be able to investigate BPS solutions of the theory and geometry of Killing spinors.

In [54] extended geometry for the group SL(N) was constructed, that may be interpreted as internal sector of a corresponding EFT. One may be interested in merging this work and the present results to end with an $\mathrm{SL}(\mathrm{N})$ "exceptional" field theory. The question is, to what extent one expect the known structures of tensor hierarchy to appear there, and is it possible to construct a Lagrangian.

\section{Acknowledgments}

The author is grateful to Ilya Bakhmatov for valuable discussions and useful comments. The author would like to thank Nesin Mathematics Village (Izmir, Turkey) and its supporters for warm hospitality during completion of a part of this research. This work on its final stage was supported by the Alexander von Humboldt Foundation.

\section{A Notations and conventions}

All the notations for indices used in this paper are as follows

$$
\begin{array}{ll}
\hat{\mathrm{M}}, \hat{\mathrm{N}}, \ldots=0, \ldots 10, & \text { 11-dimensional space-time indices; } \\
\mathrm{M}, \mathrm{N}, \ldots=0, \ldots 9, & \text { 10-dimensional space-time indices; } \\
\mu, \nu, \rho \ldots=0, \ldots 6, & \text { 7-dimensional space-time indices; } \\
\bar{a}, \bar{b}, \bar{c} \ldots=0, \ldots 6, & \text { 7-dimensional space-time flat indices; } \\
a, b, c \ldots=1, \ldots 4, & \text { 4-dimensional internal curved indices; } \\
\underline{a}, \underline{b}, \ldots=1, \ldots 4, & \text { 4-dimensional internal curved Type IIB indices; } \\
\hat{\alpha}=1,2, & \mathrm{SL}(2) \text { Type IIB index; } \\
M, N, K \ldots=1, \ldots 10, & \text { indices of the } \mathbf{1 0} \text { of } \mathrm{SL}(5) \text { labeling the extended space; } \\
m, n, k, l=1, \ldots 5, & \text { indices of the } \mathbf{5} \text { of } \mathrm{SL}(5) ; \\
\alpha, \beta=1, \ldots 4, & \text { indices of the } \mathbf{4} \text { of } \mathrm{USp}(4) ;
\end{array}
$$

The extended space of the SL(5) EFT is parametrized by the coordinates $\mathbb{X}^{M}$ with the capital Latin indices labeling the representation 10. However, it is often more convenient for explicit calculations to label the representation by an antisymmetric pair of indices in the fundamental $\mathbb{X}^{m n}=-\mathbb{X}^{m n}$. To prevent double counting one should either write sum with the condition $m<n$, or to write the $1 / 2$ prefactor explicitly. To make the calculations more straightforward and more machine-friendly we choose the second way. 
Hence, one observes the following rules to go from the capital Latin indices labeling the irrep $\mathbf{1 0}$ to an antisymmetric pair of small Latin indices each labeling the irrep $\mathbf{5}$

$$
\begin{aligned}
T^{M} & \rightarrow T^{m n} & & \text { any tensor; } \\
U^{M} V_{M} & \rightarrow \frac{1}{2} U^{m n} V_{m n} ; & & \\
\delta^{M}{ }_{N} & \rightarrow 2 \delta_{k l}^{m n} & & \text { only for the Kronecker delta. }
\end{aligned}
$$

The Kronecker delta symbol is required to be processed separately because $\delta_{m n}^{m n}=10$ as well as $\delta_{M}^{M}=10$, while one should introduce an extra $1 / 2$ factor when going from contraction of capital Latin indices to contraction of a pair of small indices.

\section{B The algebra of SL(5)}

Generators of the SL(5) group in the fundamental representation and in the representation $\mathbf{1 0}$ are given by

$$
\begin{aligned}
\left(t^{i}{ }_{j}\right)_{n}^{m} & =\delta_{j}^{m} \delta_{n}^{i}-\frac{1}{5} \delta_{n}^{m} \delta_{j}^{i}, \\
\left(t^{i}{ }_{j}\right)^{m n}{ }_{k l} & =4\left(t^{i}{ }_{j}\right)_{[k}^{[m} \delta_{l]}^{n]}
\end{aligned}
$$

These are traceless and satisfy the following commutation relations

$$
\left[t_{n}^{m}, t_{l}^{k}\right]=\delta_{l}^{m} t_{n}^{k}-\delta_{n}^{k} t_{l}^{m} .
$$

It is important to note, that when contracting generators in the $\mathbf{1 0}$ representation one should use the capital Latin indices and the same is true for the projectors below. I.e. one writes

$$
\left(t^{i} t^{k}{ }_{l}\right)^{M}{ }_{N}=\left(t_{j}^{i}\right)^{M}{ }_{K}\left(t^{k}\right)^{K}{ }_{N}=\frac{1}{2}\left(t^{i}{ }_{j}\right)^{M}{ }_{p q}\left(t^{k}{ }_{l}\right)^{p q}{ }_{N} .
$$

This results in a different coefficient in the definition of the generator in $\mathbf{1 0}$ with respect to [18], however we find such conventions more natural.

Now it is useful to write the explicit form of the projector on the $\mathbf{1 0}$ representation of $\mathrm{SL}(5)$ that reads

$$
\mathbb{P}^{M}{ }_{N}{ }^{K}{ }_{L}=\frac{1}{3}\left(t^{i}{ }_{j}\right)^{M}{ }_{N}\left(t^{i}{ }_{j}\right)^{K}{ }_{L}
$$

The identifying property of the projector then can be written as

$$
\mathbb{P}^{M}{ }_{N}{ }_{L} \mathbb{P}^{L}{ }_{K}{ }^{P} Q=\frac{1}{4} \mathbb{P}^{M}{ }_{N}{ }^{k l}{ }_{i j} \mathbb{P}^{i j}{ }_{k l}{ }^{P}{ }_{Q} \stackrel{!}{=} \mathbb{P}^{M}{ }_{N}{ }^{P} Q
$$

This fixes the overall prefactor in the projector and implies the correct identity

$$
\mathbb{P}^{M}{ }_{N}{ }^{N}{ }_{M}=\frac{1}{4} \mathbb{P}_{k l}^{m n}{ }_{k n}{ }_{m n}=24=\operatorname{dim}(\operatorname{adj}) .
$$

Let us now check explicitly the defining relation for the $Y$-tensor derived in [38], that for the SL(5) group reads

$$
\epsilon^{a M N} \epsilon_{a K L}=Y_{K L}^{M N}=-3 \mathbb{P}^{M}{ }_{N}{ }^{K}{ }_{L}+\frac{1}{5} \delta_{N}^{M} \delta_{L}^{K}+\delta_{L}^{M} \delta_{N}^{K},
$$


where $\epsilon^{a M N}$ denotes the 5-dimensional alternating symbol $\epsilon^{a m n k l}$. Taking into account the above notations we rewrite the expression as

$$
\epsilon^{a m n k l} \epsilon_{a p q r s}=-3 \mathbb{P}_{p q}^{m n}{ }_{r s}^{k l}+\frac{4}{5} \delta_{p q}^{m n} \delta_{r s}^{k l}+4 \delta_{r s}^{m n} \delta_{p q}^{k l},
$$

note the prefactor 4 of the Kronecker symbols. Substituting the expression for the projector in terms of the generators and writing them explicitly in terms of the Kronecker symbols we have for the r.h.s.

$$
\begin{aligned}
& \left(-16\left(t^{i}{ }_{j}{ }^{[m}{ }_{[p} \delta_{q]}^{n]}\left(t^{j}{ }_{i}\right)^{[k}{ }_{[r} \delta_{s]}^{l]}\right)+\frac{4}{5} \delta_{p q}^{m n} \delta_{r s}^{k l}+4 \delta_{r s}^{m n} \delta_{p q}^{k l}\right. \\
& =\left(-8 \delta_{q[r}^{m n} \delta_{s] p}^{k l}+8 \delta_{q[r}^{m n} \delta_{s] p}^{k l}+\frac{16}{5} \delta_{p q}^{m n} \delta_{r s}^{k l}\right)+\frac{4}{5} \delta_{p q}^{m n} \delta_{r s}^{k l}+4 \delta_{r s}^{m n} \delta_{p q}^{k l} \\
& =-8 \delta_{q[r}^{m n} \delta_{s] p}^{k l}+8 \delta_{q[r}^{m n} \delta_{s] p}^{k l}+4 \delta_{p q}^{m n} \delta_{r s}^{k l}+4 \delta_{r s}^{m n} \delta_{p q}^{k l}=4 ! \delta_{p q r s}^{m n k l} .
\end{aligned}
$$

This is precisely what one has on the 1.h.s. of the identity, i.e. $\epsilon^{a m n k l} \epsilon_{\text {apqrs }}=4 ! \delta_{\text {pqrs }}^{m n k l}$.

\section{Useful identities}

The 4-form field strength $\mathcal{F}^{M, K L}$ belongs to the representation $\mathbf{1 0} \otimes \overline{\mathbf{5}}=\mathbf{5}+\mathbf{4 5}$ since the indices $K L$ by construction contain only the $\overline{\mathbf{5}}$. Moreover, the representation $\mathbf{4 5}$ is not contained in the field strength. Hence, in the fundamental indices one may write $\mathcal{F}^{\text {mn },[k l r s]}$. And finally projecting out all the redundant representations one has

$$
\mathcal{F}^{m}=-6 \mathcal{F}^{m n, k l r s} \epsilon_{n k l r s} .
$$

Consider now the following expression that is relevant for the Bianchi identity of the 3-form field strength $\mathcal{F}^{K L}$

$$
\begin{aligned}
& \epsilon_{i K L}\left(\partial_{N} \mathcal{F}^{N, K L}-Y_{P Q}^{K L} \partial_{N} \mathcal{F}^{Q, P N}\right)= \\
& =\frac{1}{8}\left(\partial_{m n} \mathcal{F}^{m n, k l r s} \epsilon_{i k l r s}-6 \epsilon_{i p q r s} \partial_{m n} \mathcal{F}^{r s, p q m n}\right) .
\end{aligned}
$$

Let us show, that this is proportional to $\partial_{i j} \mathcal{F}^{j}$. Indeed, considering antisymmetrization of the indices $\{$ niklrs $\}$ in the first term, that is identically zero, and taking into account symmetries of the indices of $\mathcal{F}^{m n, k l r s}$ we may write the following

$$
\partial_{m n} \mathcal{F}^{m n, k l r s} \epsilon_{i k l r s}=\partial_{m i} \mathcal{F}^{m n, k l r s} \epsilon_{n k l r s}+4 \partial_{m k} \mathcal{F}^{m n, k l r s} \epsilon_{i n l r s} .
$$

Considering antisymmetrization of the indices $\{m n k l r s\}$ in the second term above we rewrite the above expression as

$$
\partial_{m n} \mathcal{F}^{m n, k l r s} \epsilon_{i k l r s}=\partial_{m i} \mathcal{F}^{m n, k l r s} \epsilon_{n k l r s}+\frac{1}{2} \partial_{m k} \mathcal{F}^{m k, n l r s} \epsilon_{\text {inlrs }}+3 \partial_{m k} \mathcal{F}^{n l, m k r s} \epsilon_{\text {inlrs }} .
$$

Finally, substituting back into the expression (C.2) this gives the desired identity

$$
\epsilon_{i K L}\left(\partial_{N} \mathcal{F}^{N, K L}-Y_{P Q}^{K L} \partial_{N} \mathcal{F}^{Q, P N}\right)=\frac{1}{24} \partial_{i j} \mathcal{F}^{j}
$$

The Bianchi identity itself is then written as

$$
4 \mathcal{D}_{[\mu} \mathcal{F}_{\nu \rho \sigma] i}=6 \epsilon_{i m n k l} \mathcal{F}_{[\mu \nu}^{m n} \mathcal{F}_{\rho \sigma]}^{k l}+\partial_{m i} \mathcal{F}_{\mu \nu \rho \sigma}{ }^{m} .
$$




\section{Gauge invariance of the topological Lagrangian}

In this appendix explicit check of invariance of the topological Lagrangian under all gauge transformations is provided. Let us for convenience recall the variation of the topological Lagrangian

$$
\delta \mathcal{L}_{\text {top }}=A \epsilon^{\mu \nu \rho \lambda \sigma \tau \kappa}\left[\mathcal{F}_{\mu \nu \rho \lambda}{ }^{i} \partial_{i j} \Delta C_{\sigma \tau \kappa}^{j}+6 \mathcal{F}_{\mu \nu}{ }^{i j} \mathcal{F}_{\rho \lambda \sigma i} \Delta B_{\tau \kappa j}-2 \mathcal{F}_{\mu \nu \rho i} \mathcal{F}_{\lambda \sigma \tau j} \delta A_{\kappa}^{i j}\right] .
$$

Note, that the above expression is written completely in covariant terms, while this is not true for the topological Lagrangian itself. The only way to have a covariant form is to introduce a fictitious 8-dimensional space-time with a 7-dimensional and write a covariant expression, whose variation becomes a full derivative. Hence, one obtains an integration over the 7-dimensional boundary, that is formally identified with the usual space-time.

Let us start first with generalized diffeomorphisms parametrized by $\Lambda^{M}$, that give for (D.1)

$$
\begin{aligned}
\delta_{\Lambda} \mathcal{L}_{\text {top }} \Rightarrow & \mathcal{F}_{\mu \nu \rho \lambda}{ }^{m} \partial_{m n}\left(\Lambda^{n k} \mathcal{F}_{\sigma \tau \kappa k}\right)-12 \epsilon_{n p q r s} \mathcal{F}_{\mu \nu}{ }^{m n} \mathcal{F}_{\rho \lambda \sigma m} \Lambda^{p q} \mathcal{F}_{\tau \kappa}{ }^{r s} \\
& -2 \mathcal{F}_{\mu \nu \rho m} \mathcal{F}_{\lambda \sigma \tau n} \mathcal{D}_{\kappa} \Lambda^{m n} .
\end{aligned}
$$

Here and everywhere in this section we omit the space-time alternating symbol $\epsilon^{\mu \nu \rho \lambda \sigma \tau \kappa}$ to preserve space and for clarity of notations. Hence, the corresponding antisymmetrization of all the dummy space-time indices is always undermined. In addition, since one is actually dealing with the action rather than the Lagrangian, that involves integration over the spacetime coordinates $x^{\mu}$ as well as the extended coordinates $\mathbb{X}^{m n}$, all full-derivative terms in $\mathcal{D}_{\mu}$ or $\partial_{m n}$ are dropped.

Hence, performing integration by parts in the first term with respect to $\partial_{m n}$ and in the last term with respect to $\mathcal{D}_{\mu}$ and taking into account the hidden contraction with the alternating symbol we have

$$
\begin{aligned}
& -\partial_{m n} \mathcal{F}_{\mu \nu \rho \lambda}{ }^{m} \Lambda^{n k} \mathcal{F}_{\sigma \tau \kappa k}-12 \epsilon_{n p q r s} \mathcal{F}_{\mu \nu}{ }^{m n} \mathcal{F}_{\rho \lambda \sigma m} \Lambda^{p q} \mathcal{F}_{\tau \kappa}{ }^{r s}+4\left(\mathcal{D}_{\mu} \mathcal{F}_{\nu \rho \lambda m}\right) \mathcal{F}_{\sigma \tau \kappa n} \Lambda^{m n} \\
& =6 \epsilon_{m p q r s} \mathcal{F}_{\mu \nu}{ }^{p q} \mathcal{F}_{\rho \lambda}{ }^{r s} \Lambda^{m n} \mathcal{F}_{\sigma \tau \kappa n}+12 \epsilon_{m p q r s} \mathcal{F}_{\mu \nu}{ }^{m n} \mathcal{F}_{\rho \lambda \sigma n} \Lambda^{p q} \mathcal{F}_{\tau \kappa}{ }^{r s} \\
& =18 \epsilon_{m p q r s} \mathcal{F}_{\mu \nu}{ }^{[p q} \mathcal{F}_{\rho \lambda}{ }^{r s} \Lambda^{m n]} \mathcal{F}_{\sigma \tau \kappa n} \equiv 0 .
\end{aligned}
$$

Here we have used the Bianchi identity for $\mathcal{F}_{\mu \nu \rho m}$ in the second line and organized a full antisymmetrization of six SL(5) indices $\{$ mnpqrs $\}$ labeling the $\mathbf{5}$ ensuring vanishing of the expression.

For gauge transformations parametrized by the 1-form parameter $\Xi_{\mu k}$ we write

$$
\begin{aligned}
\delta_{\Xi} \mathcal{L} \Rightarrow & 3 \mathcal{F}_{\mu \nu \rho \lambda}{ }^{m} \partial_{m n}\left(\mathcal{F}_{\sigma \tau}{ }^{n k} \Xi_{\kappa k}\right)+12 \mathcal{F}_{\mu \nu}{ }^{m n} \mathcal{F}_{\rho \lambda \sigma m} \mathcal{D}_{\tau} \Xi_{\kappa n} \\
& -\frac{1}{8} \mathcal{F}_{\mu \nu \rho m} \mathcal{F}_{\lambda \sigma \tau n} \epsilon^{m n p q r} \partial_{p q} \Xi_{\kappa r} .
\end{aligned}
$$

Performing integration by parts in all the terms and relabeling indices we obtain

$$
\begin{aligned}
& -3 \partial_{n m} \mathcal{F}_{\mu \nu \rho \lambda}{ }^{n} \mathcal{F}_{\sigma \tau}{ }^{m n} \Xi_{\kappa n}+12 \mathcal{F}_{\sigma \tau}{ }^{m n} \mathcal{D}_{\mu} \mathcal{F}_{\nu \rho \lambda m} \Xi_{\kappa n}-12 \mathcal{D}_{\tau} \mathcal{F}_{\mu \nu}{ }^{m n} \mathcal{F}_{\rho \lambda \sigma m} \Xi_{\kappa n} \\
& +\frac{1}{4} \partial_{p q} \mathcal{F}_{\mu \nu \rho m} \mathcal{F}_{\lambda \sigma \tau n} \epsilon^{m n p q r} \Xi_{\kappa r} \\
& =18 \epsilon_{m p q r s} \mathcal{F}_{\sigma \tau}{ }^{m n} \mathcal{F}_{\mu \nu}{ }^{p q} \mathcal{F}_{\rho \lambda}{ }^{r s} \Xi_{\kappa n}=18 \epsilon_{m p q r s} \mathcal{F}_{\sigma \tau}{ }^{[m n} \mathcal{F}_{\mu \nu}{ }^{p q} \mathcal{F}_{\rho \lambda}{ }^{r s]} \Xi_{\kappa n} \equiv 0 .
\end{aligned}
$$


Here the second and the last terms in the first expression cancel dues to the Bianchi identity for the 2 -form field strength $\mathcal{F}_{\mu \nu}{ }^{m n}$, while the Bianchi identity for the 3-form field strength results in a single term. Following precisely the same arguments as above one observes the indices $\{m n p q r s\}$ are fully antisymmetrized, and hence the term vanishes identically.

Finally, for the 3-form gauge transformations parametrized by $\Psi_{\mu \nu}{ }^{m}$ we have

$$
\begin{aligned}
\delta_{\Psi} \mathcal{L} & \Rightarrow 3 \mathcal{F}_{\mu \nu \rho \lambda} \partial_{m n} \mathcal{D}_{\sigma} \Psi_{\tau \kappa}{ }^{n}-6 \mathcal{F}_{\mu \nu}^{m n} \mathcal{F}_{\rho \lambda \sigma m} \partial_{k n} \Psi_{\tau \kappa}{ }^{k} \\
& =-3 \mathcal{D}_{\sigma} \mathcal{F}_{\mu \nu \rho \lambda} \partial_{m n} \Psi_{\tau \kappa}{ }^{n}-6 \mathcal{F}_{\mu \nu}^{m n} \mathcal{F}_{\rho \lambda \sigma m} \partial_{k n} \Psi_{\tau \kappa}{ }^{k} \equiv 0
\end{aligned}
$$

Here in the first line we used the fact that $\partial_{m n} \mathcal{D}_{\sigma} \Psi_{\mu \nu}{ }^{n}=\mathcal{D}_{\sigma} \partial_{m n} \Psi_{\mu \nu}{ }^{n}$ and in the second line the Bianchi identity for the 4-form field strength.

Let us now show that the used identity indeed holds, i.e. that one is allowed to swap derivatives in such expression. Effectively, this identity can be rewritten just as $\partial_{m n} \mathcal{L}_{\Lambda} \Psi^{n}-$ $\mathcal{L}_{\Lambda} \partial_{m n} \Psi^{n}=0$ for some generalized tensor $\Psi^{m}$ in the $\mathbf{5}$ of SL(5) with generalized weight $\lambda[\Psi]=3 / 5$. One notes, that this condition is nothing else but just a condition for $\partial_{m n} \Psi^{n}$ to be a generalized tensor transforming under $\overline{\mathbf{5}}$. Hence, one indeed expects this to hold as precisely this term appears in the transformation $\Delta B_{\mu \nu m}$ and in the Bianchi identity for $\mathcal{F}_{\mu \nu \rho m}$. Since all other terms in these expressions are generalized tensors the term $\partial_{m n} \Psi^{n}$ should be a generalized tensor of weight $\lambda\left[\partial_{m n} \Psi^{n}\right]=+2 / 5$.

However, let us check this explicitly and write first supposing $\partial_{m n} \Psi^{n}$ is a generalized tensor of weight $+2 / 5$

$$
\begin{aligned}
\partial_{m n} \mathcal{L}_{\Lambda} \Psi^{n}= & \frac{1}{2} \partial_{m n} \Lambda^{p q} \partial_{p q} \Psi^{n}+\frac{1}{2} \Lambda^{p q} \partial_{m n} \partial_{p q} \Psi^{n}-\frac{1}{4}\left(t^{n}{ }_{r}\right)_{p q}^{k l} \partial_{m n} \partial_{k l} \Lambda^{p q} \Psi^{r} \\
& -\frac{1}{4}\left(t^{n}{ }_{r}\right)^{k l}{ }_{p q} \partial_{k l} \Lambda^{p q} \partial_{m n} \Psi^{r}+\frac{3}{5 \cdot 2} \partial_{m n} \partial_{p q} \Lambda^{p q} \Psi^{n}+\frac{3}{5 \cdot 2} \partial_{p q} \Lambda^{p q} \partial_{m n} \Psi^{n} \\
\mathcal{L}_{\Lambda}\left(\partial_{m n} \Psi^{n}\right)= & \frac{1}{2} \Lambda^{p q} \partial_{p q} \partial_{m n} \Psi^{n}+\frac{1}{4}\left(t^{r}{ }_{m}\right)^{k l}{ }_{p q} \partial_{k l} \Lambda^{p q} \partial_{r n} \Psi^{n}+\frac{2}{5 \cdot 2} \partial_{p q} \Lambda^{p q} \partial_{m n} \Psi^{n} .
\end{aligned}
$$

We now show that these expressions are equivalent up to terms vanishing under the section condition. Taking difference of these expressions one notes, that there are terms of the form $\partial \Lambda \partial \Psi$ and $\partial \partial \Lambda \Psi$ that should vanish separately. Indeed, we have for the first type

$$
\begin{aligned}
& \Rightarrow \frac{1}{2} \partial_{m n} \Lambda^{p q} \partial_{p q} \Psi^{n}-\frac{1}{4}\left(t_{r}^{n}\right)_{p q}^{k l} \partial_{k l} \Lambda^{p q} \partial_{m n} \Psi^{r}-\frac{1}{4}\left(t_{m}^{r}\right)_{p q}^{k l} \partial_{k l} \Lambda^{p q} \partial_{r n} \Psi^{n}+\frac{1}{10} \partial_{p q} \Lambda^{p q} \partial_{m n} \Psi^{n} \\
& =\frac{1}{2} \partial_{m n} \Lambda^{p q} \partial_{p q} \Psi^{n}-\partial_{n q} \Lambda^{p q} \partial_{m p} \Psi^{n}-\partial_{m q} \Lambda^{p q} \partial_{p n} \Psi^{n}+\frac{1}{2} \partial_{p q} \Lambda^{p q} \partial_{m n} \Psi^{n} \\
& =3 \partial_{[m n} \Lambda^{p q} \partial_{p q]} \Psi^{n} \equiv 0,
\end{aligned}
$$

where in the second line the explicit form of the SL(5) generators and in the last line the section condition were used.

The similar calculation can be performed for the terms of the second type and one gets the following

$$
\begin{aligned}
& \Rightarrow-\frac{1}{4}\left(t_{r}^{n}\right)_{p q}^{k l} \partial_{m n} \partial_{k l} \Lambda^{p q} \Psi^{r}+\frac{3}{10} \partial_{m r} \partial_{p q} \Lambda^{p q} \Psi^{r} \\
& =-\partial_{m p} \partial_{r q} \Lambda^{p q} \Psi^{r}+\frac{1}{2} \partial_{m r} \partial_{p q} \Lambda^{p q} \Psi^{r}=-3 \partial_{[m p} \partial_{r q]} \Lambda^{p q} \Psi^{r} \equiv 0 .
\end{aligned}
$$


Hence, this concludes the explicit proof of invariance of the topological Lagrangian. This invariance fixes all internal coefficients, while leaving the overall prefactor arbitrary. The latter will be fixed by invariance under external 1+6-dimensional diffeomorphisms.

Open Access. This article is distributed under the terms of the Creative Commons Attribution License (CC-BY 4.0), which permits any use, distribution and reproduction in any medium, provided the original author(s) and source are credited.

\section{References}

[1] W. Siegel, Superspace duality in low-energy superstrings, Phys. Rev. D 48 (1993) 2826 [hep-th/9305073] [INSPIRE].

[2] C.M. Hull, Generalised geometry for M-theory, JHEP 07 (2007) 079 [hep-th/0701203] [INSPIRE].

[3] C. Hull and B. Zwiebach, The gauge algebra of double field theory and Courant brackets, JHEP 09 (2009) 090 [arXiv:0908.1792] [INSPIRE].

[4] D.S. Berman and M.J. Perry, Generalized geometry and M-theory, JHEP 06 (2011) 074 [arXiv: 1008.1763] [INSPIRE].

[5] D.S. Berman, H. Godazgar, M.J. Perry and P. West, Duality invariant actions and generalised geometry, JHEP 02 (2012) 108 [arXiv:1111.0459] [INSPIRE].

[6] D.S. Berman, H. Godazgar and M.J. Perry, $\mathrm{SO}(5,5)$ duality in M-theory and generalized geometry, Phys. Lett. B 700 (2011) 65 [arXiv:1103.5733] [INSPIRE].

[7] F. Hassler, D. Lüst and S. Massai, On inflation and de Sitter in non-geometric string backgrounds, arXiv:1405.2325 [INSPIRE].

[8] R. Blumenhagen, A. Font and E. Plauschinn, Relating double field theory to the scalar potential of $N=2$ gauged supergravity, JHEP 12 (2015) 122 [arXiv:1507.08059] [INSPIRE].

[9] G. Dibitetto, A. Guarino and D. Roest, Exceptional flux compactifications, JHEP 05 (2012) 056 [arXiv: 1202.0770] [INSPIRE].

[10] G. Dibitetto, J.J. Fernandez-Melgarejo, D. Marques and D. Roest, Duality orbits of non-geometric fluxes, Fortsch. Phys. 60 (2012) 1123 [arXiv:1203.6562] [INSPIRE].

[11] F. Hassler and D. Lüst, Non-commutative/non-associative IIA (IIB) Q-and R-branes and their intersections, JHEP 07 (2013) 048 [arXiv: 1303.1413] [INSPIRE].

[12] S. Jensen, The KK-monopole/NS5-brane in doubled geometry, JHEP 07 (2011) 088 [arXiv: 1106.1174] [INSPIRE].

[13] D. Andriot, M. Larfors, D. Lüst and P. Patalong, A ten-dimensional action for non-geometric fluxes, JHEP 09 (2011) 134 [arXiv:1106.4015] [INSPIRE].

[14] D. Andriot, O. Hohm, M. Larfors, D. Lüst and P. Patalong, Non-geometric fluxes in supergravity and double field theory, Fortsch. Phys. 60 (2012) 1150 [arXiv:1204.1979] [INSPIRE].

[15] D. Andriot, O. Hohm, M. Larfors, D. Lüst and P. Patalong, A geometric action for non-geometric fluxes, Phys. Rev. Lett. 108 (2012) 261602 [arXiv:1202.3060] [INSPIRE].

[16] K. Lee, C. Strickland-Constable and D. Waldram, New gaugings and non-geometry, arXiv: 1506.03457 [INSPIRE]. 
[17] M. Graña and D. Marqués, Gauged double field theory, JHEP 04 (2012) 020 [arXiv: 1201.2924] [INSPIRE].

[18] D.S. Berman, E.T. Musaev, D.C. Thompson and D.C. Thompson, Duality invariant M-theory: gauged supergravities and Scherk-Schwarz reductions, JHEP 10 (2012) 174 [arXiv:1208.0020] [INSPIRE].

[19] E.T. Musaev, Gauged supergravities in 5 and 6 dimensions from generalised Scherk-Schwarz reductions, JHEP 05 (2013) 161 [arXiv:1301.0467] [INSPIRE].

[20] C.D.A. Blair, E. Malek and J.-H. Park, M-theory and type IIB from a duality manifest action, JHEP 01 (2014) 172 [arXiv:1311.5109] [INSPIRE].

[21] W.H. Baron, Gaugings from $E_{7(7)}$ extended geometries, Phys. Rev. D 91 (2015) 024008 [arXiv: 1404.7750] [INSPIRE].

[22] E. Malek and H. Samtleben, Dualising consistent IIA/IIB truncations, JHEP 12 (2015) 029 [arXiv: 1510.03433] [INSPIRE].

[23] A. Baguet, O. Hohm and H. Samtleben, Consistent type IIB reductions to maximal $5 D$ supergravity, Phys. Rev. D 92 (2015) 065004 [arXiv:1506.01385] [INSPIRE].

[24] A. Baguet, O. Hohm and H. Samtleben, $E_{6(6)}$ exceptional field theory: review and embedding of type IIB, PoS (CORFU2014) 133 [arXiv: 1506.01065] [INSPIRE].

[25] A. Coimbra, C. Strickland-Constable and D. Waldram, $E_{d(d)} \times \mathbb{R}^{+}$generalised geometry, connections and M-theory, JHEP 02 (2014) 054 [arXiv:1112.3989] [INSPIRE].

[26] A. Coimbra, C. Strickland-Constable and D. Waldram, Supergravity as generalised geometry I: type II theories, JHEP 11 (2011) 091 [arXiv:1107.1733] [INSPIRE].

[27] A. Coimbra, C. Strickland-Constable and D. Waldram, Supergravity as generalised geometry II: $E_{d(d)} \times \mathbb{R}^{+}$and M-theory, JHEP 03 (2014) 019 [arXiv: 1212.1586] [INSPIRE].

[28] A. Coimbra, C. Strickland-Constable and D. Waldram, Supersymmetric backgrounds and generalised special holonomy, arXiv:1411.5721 [INSPIRE].

[29] M. Cederwall, T-duality and non-geometric solutions from double geometry, Fortsch. Phys. 62 (2014) 942 [arXiv:1409.4463] [INSPIRE].

[30] D.S. Berman, M. Cederwall and M.J. Perry, Global aspects of double geometry, JHEP 09 (2014) 066 [arXiv: 1401.1311] [INSPIRE].

[31] M. Cederwall, The geometry behind double geometry, JHEP 09 (2014) 070 [arXiv: 1402.2513] [INSPIRE].

[32] S.-J. Rey and Y. Sakatani, Finite transformations in doubled and exceptional space, arXiv: 1510.06735 [INSPIRE].

[33] J. Palmkvist, Exceptional geometry and Borcherds superalgebras, JHEP 11 (2015) 032 [arXiv: 1507.08828] [INSPIRE].

[34] I. Bandos, Superstring in doubled superspace, Phys. Lett. B 751 (2015) 408 [arXiv: 1507.07779] [INSPIRE].

[35] D.S. Berman and F.J. Rudolph, Strings, branes and the self-dual solutions of exceptional field theory, JHEP 05 (2015) 130 [arXiv: 1412.2768] [INSPIRE].

[36] D.S. Berman and F.J. Rudolph, Branes are waves and monopoles, JHEP 05 (2015) 015 [arXiv: 1409.6314] [INSPIRE]. 
[37] J. Berkeley, D.S. Berman and F.J. Rudolph, Strings and branes are waves, JHEP 06 (2014) 006 [arXiv: 1403.7198] [INSPIRE].

[38] D.S. Berman, M. Cederwall, A. Kleinschmidt and D.C. Thompson, The gauge structure of generalised diffeomorphisms, JHEP 01 (2013) 064 [arXiv:1208.5884] [INSPIRE].

[39] G. Aldazabal, D. Marqués and C. Núñez, Double field theory: a pedagogical review, Class. Quant. Grav. 30 (2013) 163001 [arXiv:1305.1907] [INSPIRE].

[40] D.S. Berman and D.C. Thompson, Duality symmetric string and M-theory, Phys. Rept. 566 (2014) 1 [arXiv:1306.2643] [InSPIRE].

[41] O. Hohm, D. Lüst and B. Zwiebach, The spacetime of double field theory: review, remarks and outlook, Fortsch. Phys. 61 (2013) 926 [arXiv:1309.2977] [INSPIRE].

[42] O. Hohm and H. Samtleben, U-duality covariant gravity, JHEP 09 (2013) 080 [arXiv: 1307.0509] [INSPIRE].

[43] O. Hohm and H. Samtleben, Exceptional form of $D=11$ supergravity, Phys. Rev. Lett. 111 (2013) 231601 [arXiv:1308.1673] [inSPIRE].

[44] O. Hohm and H. Samtleben, Exceptional field theory III. E $E_{8(8)}$, Phys. Rev. D 90 (2014) 066002 [arXiv:1406.3348] [INSPIRE].

[45] O. Hohm and H. Samtleben, Exceptional field theory II. $E_{7(7)}$, Phys. Rev. D 89 (2014) 066017 [arXiv:1312.4542] [INSPIRE].

[46] O. Hohm and H. Samtleben, Exceptional field theory I. $E_{6(6)}$ covariant form of M-theory and type IIB, Phys. Rev. D 89 (2014) 066016 [arXiv:1312.0614] [InSPIRE].

[47] A. Abzalov, I. Bakhmatov and E.T. Musaev, Exceptional field theory: $\mathrm{SO}(5,5)$, JHEP 06 (2015) 088 [arXiv: 1504.01523] [INSPIRE].

[48] O. Hohm and Y.-N. Wang, Tensor hierarchy and generalized Cartan calculus in $\mathrm{SL}(3) \times \mathrm{SL}(2)$ exceptional field theory, JHEP 04 (2015) 050 [arXiv: 1501.01600] [INSPIRE].

[49] H. Godazgar, M. Godazgar, O. Hohm, H. Nicolai and H. Samtleben, Supersymmetric $E_{7(7)}$ exceptional field theory, JHEP 09 (2014) 044 [arXiv:1406.3235] [INSPIRE].

[50] E. Musaev and H. Samtleben, Fermions and supersymmetry in $E_{6(6)}$ exceptional field theory, JHEP 03 (2015) 027 [arXiv:1412.7286] [INSPIRE].

[51] E.T. Musaev, Exceptional field theory for $E_{6(6)}$ supergravity, TSPU Bulletin 12 (2014) 198 [arXiv: 1503.08397] [INSPIRE].

[52] D.S. Berman, E.T. Musaev and M.J. Perry, Boundary terms in generalized geometry and doubled field theory, Phys. Lett. B 706 (2011) 228 [arXiv:1110.3097] [InSPIRE].

[53] J. Berkeley, Solution-generating transformations in duality-invariant theories and the fluid/gravity correspondence, Ph.D. thesis, Queen Mary U., London U.K. (2015) [arXiv: 1511.00995] [INSPIRE].

[54] J.-H. Park and Y. Suh, U-gravity: SL(N), JHEP 06 (2014) 102 [arXiv:1402.5027] [INSPIRE].

[55] E. Sezgin and A. Salam, Maximal extended supergravity theory in seven-dimensions, Phys. Lett. B 118 (1982) 359 [INSPIRE].

[56] E. Cremmer, B. Julia, H. Lü and C.N. Pope, Dualization of dualities. 1, Nucl. Phys. B 523 (1998) 73 [hep-th/9710119] [INSPIRE]. 
[57] H. Samtleben and M. Weidner, The maximal $D=7$ supergravities, Nucl. Phys. B 725 (2005) 383 [hep-th/0506237] [inSPIRE].

[58] D.C. Thompson, Duality invariance: from M-theory to double field theory, JHEP 08 (2011) 125 [arXiv: 1106.4036] [INSPIRE].

[59] R. Blumenhagen, F. Hassler and D. Lüst, Double field theory on group manifolds, JHEP 02 (2015) 001 [arXiv: 1410.6374] [INSPIRE].

[60] P.d. Bosque, F. Hassler and D. Lüst, Flux formulation of DFT on group manifolds and generalized Scherk-Schwarz compactifications, arXiv:1509.04176 [INSPIRE].

[61] R. Blumenhagen, P.d. Bosque, F. Hassler and D. Lüst, Generalized metric formulation of double field theory on group manifolds, JHEP 08 (2015) 056 [arXiv: 1502.02428] [INSPIRE].

[62] A. Baguet, C.N. Pope and H. Samtleben, Consistent Pauli reduction on group manifolds, Phys. Lett. B 752 (2016) 278 [arXiv:1510.08926] [InSPIRE]. 